\title{
The circular RNA ACR attenuates myocardial ischemia/reperfusion injury by suppressing autophagy via modulation of the Pink1/ FAM65B pathway
}

\author{
Lu-Yu Zhou ${ }^{1} \cdot$ Mei Zhai ${ }^{2} \cdot$ Yan Huang ${ }^{2} \cdot$ Sheng $\mathrm{Xu}^{1} \cdot$ Tao $\mathrm{An}^{2} \cdot$ Yun-Hong Wang ${ }^{2} \cdot$ Rong-Cheng Zhang $^{2} \cdot$ \\ Cui-Yun Liu ${ }^{1}$ Y Yan-Han Dong ${ }^{1}$ - Man Wang ${ }^{1} \cdot$ Li-Li Qian $^{1} \cdot$ Murugavel Ponnusamy ${ }^{1} \cdot$ Yu-Hui Zhang ${ }^{2} \cdot J_{i a n}$ Zhang $^{2}$. \\ Kun Wang ${ }^{1}$
}

Received: 9 April 2018 / Revised: 30 August 2018 / Accepted: 6 September 2018 / Published online: 22 October 2018

(c) ADMC Associazione Differenziamento e Morte Cellulare 2018

\begin{abstract}
Dysregulated autophagy is associated with many pathological disorders such as cardiovascular diseases. Emerging evidence has suggested that circular RNAs (circRNAs) have important roles in some biological processes. However, it remains unclear whether circRNAs participate in the regulation of autophagy. Here we report that a circRNA, termed autophagyrelated circular RNA (ACR), represses autophagy and myocardial infarction by targeting Pink1-mediated phosphorylation of FAM65B. ACR attenuates autophagy and cell death in cardiomyocytes. Moreover, ACR protects the heart from ischemia/ reperfusion (I/R) injury and reduces myocardial infarct sizes. We identify Pink1 as an ACR target to mediate the function of ACR in cardiomyocyte autophagy. ACR activates Pink1 expression through directly binding to Dnmt3B and blocking Dnmt3B-mediated DNA methylation of Pink1 promoter. Pink1 suppresses autophagy and Pink1 transgenic mice show reduced myocardial infarction sizes. Further, we find that FAM65B is a downstream target of Pink1 and Pink1 phosphorylates FAM65B at serine 46. Phosphorylated FAM65B inhibits autophagy and cell death in the heart. Our findings reveal a novel role for the circRNA in regulating autophagy and ACR-Pink1-FAM65B axis as a regulator of autophagy in the heart will be potential therapeutic targets in treatment of cardiovascular diseases.
\end{abstract}

These authors contributed equally: Lu-Yu Zhou, Mei Zhai, Yan Huang, and Sheng Xu

Edited by H.-U. Simon

Electronic supplementary material The online version of this article (https://doi.org/10.1038/s41418-018-0206-4) contains supplementary material, which is available to authorized users.

Kun Wang

wangk696@qdu.edu.cn

1 Center for Developmental Cardiology, Institute for Translational Medicine, College of Medicine, Qingdao University,

Qingdao 266021, China

2 State Key Laboratory of Cardiovascular Disease, Heart Failure Center, Fuwai Hospital, National Center for Cardiovascular Diseases, Chinese Academy of Medical Sciences, Peking Union Medical College, Beijing 100037, China

\section{Introduction}

Autophagy is a well-established conserved mechanism that delivers the intracellular constituents and organelles to lysosomes for degradation [1]. More and more studies suggest that autophagic death is an important process that is distinct from apoptosis [2]. Autophagy has been demonstrated to have a critical role in many physiological and pathological processes. Dysregulation of autophagy is associated with a number of cardiac diseases including dilated cardiomyopathy, ischemic heart disease, and heart failure [3-5]. Although the regulation of autophagy is important in cardiovascular diseases, there is no effective treatment for the autophagy-related cardiac diseases and heart failure. Exploring and revealing the molecular mechanisms underlying the regulation of autophagy will provide a potential interventional strategy for treating cardiovascular diseases.

Circular RNA (circRNA) is a class of covalently closed, single-stranded non-coding RNAs that are transcribed by RNA polymerase II [6]. CircRNA are widely expressed in flies, worms, mouse, and human [6-8]. CircRNAs have 
been identified in several cell lines and exert important functions. Deletion of the circular transcripts while maintaining the expression of the linear RNA isoforms results in renal aplasia [9]. CDR1as, also called CiRS-7, is a highly stable and conserved circRNA that is expressed in both human and mouse brain $[6,10]$. The overexpression of CDR1as results in impairment of midbrain development [10]. The circRNA that derived from sex-determining region $\mathrm{Y}$ functions as a molecular sponge that contains 16 binding sites for miR-138 and inhibits the activity of miR138 [10]. The circRNA cZNF292 contains no binding site for miRNA, is abundantly expressed in endothelial cells, and has proangiogenic functions [11]. Besides, the circRNA of the virusoid contains internal ribosome entry site and exhibits coding potential [12]. Hitherto, some studies suggest that circRNAs have important functions in many cellular processes. However, the role of circRNA in autophagy remains unclear and awaits future studies.

Phosphatase and tensin homolog-induced putative kinase 1 (Pink1) is a conserved mitochondrial serine/threonine protein kinase that localizes throughout the cytosol [13]. Pink1 targets to the outer membrane of mitochondria and can be imported into mitochondrial matrix as well [13-15]. Pink1 ensures the quality control of mitochondria and is indispensable for maintaining the normal morphology of mitochondria [16]. The serine/threonine kinase domain of Pink1 has a key role in regulating its downstream targets through phosphorylation [17]. Miro, ubiquitin, and Rab GTPases are phosphorylated by Pink1 [18-21]. In addition, The tumor necrosis factor type1 receptor-associated protein, the serine protease HtrA2/Omi, PGC1 $\alpha$ regulator PARIS, and mitochondrial complex I subunit NdufA10 are also potential Pink1 substrates [22-25]. Mutations in PINK1 kinase domain are found in a number of Parkinson's disease patients [26]. Pink1 is expressed in the heart, muscles, and testes [27]. It is of note that Pink1 is downregulated during human end-stage heart failure, which suggests that Pink1 is essential for normal heart function [28]. Previous studies suggest that Pink1 is involved in many pathological and physiological processes. However, it remains largely unknown whether Pink1 can regulate autophagy in the heart and its downstream targets need to be further identified.

Family with sequence similarity 65 member B (FAM65B) is an atypical inhibitor of the small $\mathrm{G}$ protein RhoA and is transcriptionally regulated by FOXO1 [29]. FAM65B is involved in many cellular processes, including cell adhesion, polarization, migration, and differentiation [30-32]. FAM65B overexpression blocks mitosis in the transformed T cells and decrease of FAM65B expression is required for proliferation [33]. Fam65B functions in neutrophil polarization and abnormalities in FAM65B results in deficiency in directional chemotaxis [34]. Although these studies suggest that FAM65B has a pivotal role in some biological processes, the function of FAM65B in cardiomyocytes remains unclear. Furthermore, it is not yet clear whether FAM65B can regulate autophagic program.

Our present study reveals that circRNA ACR (autophagy-related circRNA) participates in the regulation of cardiomyocyte autophagy and myocardial infarction (INF). ACR represses autophagic cell death and attenuates myocardial INF sizes. Next, we find that Pink1 can act as a downstream target of ACR to mediate autophagy and myocardial INF. ACR regulates Pink1 expression through directly binding to Dnmt3B and affecting Dnmt3Bmediated DNA methylation of Pink1 promoter. Moreover, our study further demonstrates that FAM65B can be phosphorylated by Pink1 at serine 46, and phosphorylated FAM65B exerts an inhibitory effect on autophagy and cell death in the heart. Our findings reveal a novel regulation function of circRNA in autophagy and also identify a new molecular mechanism of circRNA in the treatment of cardiovascular diseases.

\section{Methods}

\section{Cardiomyocytes culture and treatment}

Cardiomyocytes were isolated from 1 to 2 days old mice as we described [35]. Briefly, after dissection hearts were washed and minced in HEPES-buffered saline solution. Tissues were then dispersed in a series of incubations at $37^{\circ} \mathrm{C}$ in HEPES-buffered saline solution containing 1.2 $\mathrm{mg} / \mathrm{ml}$ pancreatin and $0.14 \mathrm{mg} / \mathrm{ml}$ collagenase. Subsequent supernatants were collected and centrifuged at $200 \times g$ for $5 \mathrm{~min}$. After centrifugation, cells were resuspended in Dulbecco's modified Eagle's medium/F-12 (GIBCO) containing 5\% heat-inactivated Fetal bovine serum, $0.1 \mathrm{mM}$ ascorbate, insulin-transferring-sodium selenite media supplement (Sigma, St. Louis, MO), 100 $\mathrm{U} / \mathrm{ml}$ penicillin, $100 \mu \mathrm{g} / \mathrm{ml}$ streptomycin, and $0.1 \mathrm{mM}$ bromodeoxyuridine. The dissociated cells were pre-plated at $37^{\circ} \mathrm{C}$ for $1 \mathrm{~h}$. The cells were then diluted to $1 \times 10^{6}$ cells $/ \mathrm{ml}$ and plated in $10 \mu \mathrm{g} / \mathrm{ml}$ laminin-coated different culture dishes according to the specific experimental requirements. For anoxia/re-oxygenation (A/R) performance, cardiomyocytes were placed in an anoxic chamber with a water-saturated atmosphere comprising $5 \% \mathrm{CO}_{2}$ and $95 \% \mathrm{~N}_{2}$. After anoxia, the cells were subjected to reoxygenation $\left(95 \% \mathrm{O}_{2}\right.$ and $\left.5 \% \mathrm{CO}_{2}\right)$.

\section{Cell death assay}

Cell death was determined by Trypan Blue exclusion, and the numbers of Trypan Blue-positive and Trypan Bluenegative cells were counted on a hemocytometer. 


\section{siRNA constructions of ACR, Pink1, and FAM65B}

ACR is knocked down using customdesigned small interfering RNA (siRNA) oligonucleotides. ACR RNA interference (RNAi) target sequence is $5^{\prime}$-AGAAAAAATAG GATAGCAGCAG-3'. A non-related, scrambled RNAi sequence is used as a control (5'-AAGAAGTCAAGTAC AGAGAAGA-3'). The mouse Pinkl RNAi target sequence is $5^{\prime}$-CCAAGCGCGTGTCTGACCC-3'. A scramble form was used as a control (5'-AGCCGTCGACTGCACTCGC$\left.3^{\prime}\right)$. The mouse FAM65B RNAi target sequence is $5^{\prime}$-GG ACAAATTGACTGCTCAA-3'. A non-related, scrambled RNAi sequence is used as a control (5'-GCACCTAGGC GAATTATAA- $3^{\prime}$ ). The adenoviruses harboring these RNAi constructs are generated using the pSilencer ${ }^{\mathrm{TM}}$ adeno 1.0-CMV System (Ambion) according to the manufacturer's instructions.

\section{Adenoviral constructions and infection}

CircRNA ACR vector was synthesized as previous studies described $[10,36]$. We inserted the ACR exon along with the endogenous flanking sequence (1 kb upstream) into pcDNA3.1. Then we copied part of the upstream flanking sequence and inserted it in an inverted orientation downstream. ACR-ir without the downstream reverse sequence was used as negative control. The mouse coding sequences of Pink1, FAM65B-wt, and FAM65B-46A were synthesized by PCR using mouse cDNA as the template. The adenoviral constructs were prepared using the Adeno- $\mathrm{X}^{\mathrm{TM}}$ Expression System (Clontech) according to the manufacturer's instructions.

\section{Immunoblotting}

Immunoblotting was performed as we described [37]. The cells were lysed for $1 \mathrm{~h}$ at $4{ }^{\circ} \mathrm{C}$ in a lysis buffer $(20 \mathrm{mmol} / \mathrm{L}$ Tris $\mathrm{pH}$ 7.5, $2 \mathrm{mmol} / \mathrm{L}$ EDTA, $3 \mathrm{mmol} / \mathrm{L}$ EGTA, $2 \mathrm{mmol} / \mathrm{L}$ dithiothreitol (DTT), $250 \mathrm{mmol} / \mathrm{L}$ sucrose, $0.1 \mathrm{mmol} / \mathrm{L}$ phenylmethylsulfonyl fluoride, $1 \%$ Triton X-100) containing a protease inhibitor cocktail. The samples were subjected to $10 \%$ or $12 \%$ SDS-polyacrylamide gel electrophoresis (PAGE) and transferred to nitrocellulose membranes. Equal protein loading was controlled by Ponceau Red staining of membranes. Blots were probed using the primary antibodies. The anti-Pink1 antibody (1:500), anti-LC3 antibody (1:500), and anti-Actin antibody (1:2000) were from Abcam. The anti-FAM65B (1:500) antibody was from Mybiosource. After four times washing with phosphate-buffered saline (PBS), the horseradish peroxidase-conjugated secondary antibodies were added. Antigen-antibody complexes were visualized by enhanced chemiluminescence.

\section{Quantitative reverse transcription-PCR}

Divergent primers were designed for ACR. The sequences of ACR primers were forward 5'-GAAGTTGCTTTATG TTCTGG-3' and reverse 5'-TGTCTGGAGTTCTTCAA AGG-3'. The sequences of Pink1 primers were forward 5'ATCCAGAGATGGTCCCAAGCA-3' and reverse 5'CATGGTAGCCAGGCGAGAA- $3^{\prime}$. The results were standardized to control values of glyceraldehyde-3-phosphate dehydrogenase (GAPDH). The sequences of GAPDH primers were forward 5'-TGTGTCCGTCGTGGATCTG A- $3^{\prime}$ and reverse 5'-CCTGCTTCACCACCTTCTTG A-3'. The specificity of the PCR amplification was confirmed by agarose gel electrophoresis.

\section{RNA-binding protein immunoprecipitation assay}

RNA-binding protein immunoprecipitation (RIP) was performed using a Magna RIP RNA-Binding Protein Immunoprecipitation Kit (Millipore). Briefly, cardiomyocytes were collected by adding RIP lysis buffer and incubated with protein beads and antibody complex overnight at $4{ }^{\circ} \mathrm{C}$. After washing, RNAs binding to protein were eluted and quantified. Quantitative reverse transcription-PCR (qRTPCR) was performed to examine certain RNAs coimmunoprecipitated with the antibody.

\section{Pull-down assay with biotinylated DNA probe}

The biotinylated DNA probe complementary to ACR RNA was synthesized and dissolved in $500 \mu \mathrm{l}$ of wash/binding buffer $(0.5 \mathrm{M} \mathrm{NaCl}, 20 \mathrm{mM}$ Tris- $\mathrm{HCl} \mathrm{pH} 7.5$, and $1 \mathrm{mM}$ EDTA). The probes were incubated with streptavidincoated magnetic beads (Sigma) at $25^{\circ} \mathrm{C}$ for $2 \mathrm{~h}$ to generate probe-coated magnetic beads. Cardiomyocytes protein extract were incubated with probe-coated beads for $2 \mathrm{~h}$. After washing with the wash/binding buffer, the retrieved protein was subjected to western blot analysis. The following probe sequences were used: ACR pull-down probe 5'-CTGCTGCTGCTATCCTATTTTTTCTTGCCC-3' and random pull-down probe $5^{\prime}$-TGATGTCTAGCGCTTGGG CTTTG-3'.

\section{Chromatin immunoprecipitation assay}

Chromatin immunoprecipitation (ChIP) assay was performed as we described [38]. In brief, cells were washed with PBS and incubated for 10 min with $1 \%$ formaldehyde at room temperature. The cross-linking was quenched with $0.1 \mathrm{M}$ glycine for $5 \mathrm{~min}$. Cells were washed twice with PBS and lysed for $1 \mathrm{~h}$ at $4{ }^{\circ} \mathrm{C}$ in a lysis buffer. The cell lysates were sonicated into chromatin fragments with an average length of $500-800 \mathrm{bp}$ as assessed by agarose gel 
electrophoresis. The samples were precleared with ProteinA agarose (Roche) for $1 \mathrm{~h}$ at $4{ }^{\circ} \mathrm{C}$ on a rocking platform and $5 \mu \mathrm{g}$ specific antibodies were added and rocked for overnight at $4{ }^{\circ} \mathrm{C}$. Immunoprecipitates were captured with $10 \%$ (vol/vol) Protein-A agarose for $4 \mathrm{~h}$. Before use, Protein-A agarose was blocked twice at $4{ }^{\circ} \mathrm{C}$ with salmon sperm DNA $(2 \mu \mathrm{g} / \mathrm{ml})$ overnight. DNA fragments were purified with a QIAquick Spin Kit (Qiagen). The purified DNA was used as a template and amplified with the following primer sets: 5'-ACCATGAACTGCCTCTGGAT-3' and 5'-TTGTTGT CCCAGACGTTTGTG-3'.

\section{Methylation detection}

Bisulfite conversion reactions were performed using an EZ DNA Methylation-Gold kit (ZYMO Research, Orange, CA) according to the manufacturer's instructions. Bisulfitetreated DNA was cloned into pGEM-T (Promega) and, for each group, 20 clones were sequenced to analyze the Pink1 promoter from -61 to +546 relative to the transcription start site.

\section{In vitro kinase assay}

FAM65B cDNA was constructed into pet-28a vector (Novagen) and expressed in Rosetta bacteria (Novagen). Recombinant FAM65B-His protein was purified by NiNTA His $\bullet$ Bind ${ }^{\circledR}$ Resin (Merck Millipore) using the manufacturer's protocol. Pink1-cMyc was immunoprecipitated by the anti-cMyc antibody from cells and added to the FAM65B-His protein with modified kinase buffer [21] (50 mM Tris- $\mathrm{HCl}, 10 \mathrm{mM} \mathrm{MgCl}_{2}, 5 \mathrm{mM}$ DTT, $400 \mu \mathrm{M}$ ATP, $\left.5 \mathrm{mM} \mathrm{NaF}, 0.5 \mathrm{mM} \mathrm{Na} 3 \mathrm{VO}_{4} \mathrm{pH} 7.5\right)$. The reaction was for $2 \mathrm{~h}$ at $30^{\circ} \mathrm{C}$ and analyzed by immunoblotting.

\section{Generation of phospho-antibodies}

The phospho-Serine-46 (S46) antibody was produced by SBS Genetech Co., Ltd. The phospho-T119 antibody and phospho-S558 antibody were produced by BeiJing Cowin Biotech Co., Ltd. The phosphopeptides SQSpFAGFSGLQER (amino acids 44-53), DEYLEFHQTpELDKLC (amino acids 111-124), and LQESDEASpELKPVEC (amino acids 551-564) were used to immunize the rabbits to prepare polyclonal antibodies against FAM65B. The antiserum was purified to deplete antibodies that recognize nonphosphorylated FAM65B.

\section{Reticulocytes lysate system}

Pink1, FAM65B-wt, and its mutants were translated using the $\mathrm{TNT}^{\circledR} \mathrm{T} 7$ coupled reticulocytes lysate system (Promega) according to the manufacturer's instructions. pcDNA3.1 is the control empty plasmid. FAM65B mutants were generated using the QuikChange ${ }^{\circledast}$ Site-Directed Mutagenesis Kit (Invitrogen).

\section{Samples preparation for mass spectrometry}

Protein samples were separated by $6 \%$ SDS-PAGE and staining with Coomassie Brilliant Blue. The gel bands of interest were manually excised. Each of the protein bands was digested separately. Then the sliced gels were cut into small plugs, washed twice in $200 \mu \mathrm{l}$ of distilled water for 10 min, and destained as previously described [39]. The gel bands were dehydrated in $100 \%$ acetonitrile for $10 \mathrm{~min}$ and dried in the Speedvac (Labconco) for approximately 15 min. Reduction and alkylation were performed as previously reported [40], followed by washing of the gel plugs with $50 \%$ acetonitrile in $25 \mathrm{mM}$ ammonium bicarbonate twice. The gel plugs were then dried using the speedvac and digested with sequence-grade modified trypsin $(40 \mathrm{ng}$ for each band) in $25 \mathrm{mM} \mathrm{NH} \mathrm{HCO}_{3}$ overnight at $37^{\circ} \mathrm{C}$. The enzymatic reaction was stopped by adding $1 \%$ formic acid. The solution was then transferred to a sample vial for liquid chromatography/tandem mass spectrometry (LC-MS/MS) analysis.

\section{Electrospray ionization ion trap MS analysis}

LC-MS/MS analysis was performed using a Thermo Fisher Finnigan LTQ linear ion trap mass spectrometer in line with a Thermo Fisher Finnigan Surveyor MS Pump Plus HPLC system. Tryptic peptides generated above were loaded onto a trap column (300SB-C18, $5 \times 0.3 \mathrm{~mm}, 5 \mu \mathrm{m}$ particle) (Agilent Technologies, Santa Clara CA), which was connected through a zero dead volume union to the self-packed analytical column $(\mathrm{C} 18,100 \mu \mathrm{m}$ i.d. $\times 100 \mathrm{~mm}, 3 \mu \mathrm{m}$ particle) (SunChrom, Germany). The peptides were then eluted over a gradient $(0-45 \% B$ in $55 \mathrm{~min}, 45-100 \% B$ in $10 \mathrm{~min}$, where $B=80 \%$ acetonitrile, $0.1 \%$ formic acid) at a flow rate of $500 \mathrm{~nL} / \mathrm{min}$ and introduced online into the linear ion trap mass spectrometer (Thermo Fisher Corporation, San Jose, CA) using nanoelectrospray ionization. Data-dependent scanning was incorporated to select the five most abundant ions (one microscan per spectra; precursor isolation width $1.0 \mathrm{~m} / \mathrm{z}, 35 \%$ collision energy, $30 \mathrm{~ms}$ ion activation, exclusion duration: $90 \mathrm{~s}$; repeat count: 1) from a full-scan mass spectrum for fragmentation by collision-induced dissociation.

\section{Microarray analysis}

For circRNA microarrays, total RNAs were extracted from the sham-treated mice and I/R-treated mouse heart tissues. The fluorescent cRNA was obtained using random primers 
according to Arraystar Super RNA Labeling protocol (Arraystar Super RNA Labeling Kit; Arraystar). Then the labeled cDNA was hybridized to Arraystar mouse circRNA Array. The microarray hybridization and the collection of data were performed by Kang Chen Bio-tech, Shanghai, China. For the transcriptome microarray, total RNA was extracted from cardiomyocytes by using Trizol reagent and then the isolated mRNAs were reverse transcribed and hybridized to mouse Genome Array (CapitalBio Corp.).

\section{Transmission electron microscopy}

Conventional electron microscopy was performed as described previously [41]. In brief, cells were fixed with $2.5 \%$ glutaraldehyde and then postfixed with $1 \%$ osmium tetraoxide, dehydrated in a graded series of ethanol concentrations, and embedded in Embed812 resin. The ultrathin sections were mounted on copper grids and then double-stained with uranyl acetate and lead citrate. The number of autophagic vacuoles was determined for a minimum of 100 cells. Heart ultrastructural analysis was also performed. The samples were examined and photographed with a FEI Tecnai spirit transmission electron microscope.

\section{Echocardiographic assessment}

Transthoracic echocardiographic analysis was performed on mice after the sham or $\mathrm{I} / \mathrm{R}$ surgery as we described [42]. Echocardiographic parameters were measured. Fractional shortening of left ventricular diameter was calculated as [(LVIDd - LVIDs)/LVIDd] $\times 100$. After in vivo evaluation of cardiac function, the mice were killed and the hearts were collected, weighted, and used for histological examination.

\section{Animal experiments}

Male adult C57BL/6 mice (10 weeks old) were obtained from Institute of Laboratory Animal Science of Chinese Academy of Medical Sciences (Beijing, China). All experiments were performed according to the protocols approved by the Animal Care Committee, Qingdao University. For intracoronary delivery of adenoviruses, the mice were anesthetized and ventilated with a HX-300S animal ventilator. The chest was entered through a small left anterior thoracotomy, the pericardial sac was then removed, and $2 \times 10^{11}$ adenoviruses of ACR-siRNA were injected with a catheter from the apex of the left ventricle (LV) into the aortic root, while the aorta and pulmonary arteries were cross-clamped. The clamp was maintained for $20 \mathrm{~s}$ when the heart pumped against a closed system. After removal of air and blood, the chest was then closed and the mice were returned back to cage for recovery. Five days after the injection of adenoviruses, the mice were subjected to $I / R$ surgery.

For I/R injury model, Pink1 transgenic mice and wildtype (WT) mice were subjected to $60 \mathrm{~min}$ ischemia, then $3 \mathrm{~h}$ or 1 week reperfusion as we described [43]. Sham-operated group experienced the same procedure, except the snare was left untied. After $3 \mathrm{~h}$ of reperfusion, Evans Blue dye $(1 \mathrm{ml}$ of a $2.0 \%$ solution; Sigma-Aldrich) was injected into the jugular vein into the heart for delineation of the ischemic zone from the non-ischemic zone. The heart was rapidly excised. The heart slices were incubated in 1.0\% 2,3,5-triphenyltetrazolium chloride (Sigma-Aldrich) for $15 \mathrm{~min}$ at $37^{\circ} \mathrm{C}$ for demarcation of the viable and non-viable myocardium within the risk zone. The staining was stopped by ice-cold sterile saline and the slices were fixed in $10 \%$ neutral buffered formaldehyde and individually weighed. Both sides of each slice were photographed. The areas of INF and non-ischemic LV were assessed with computerassisted planimetry (NIH Image 1.57) by an observer blinded to the sample identity.

\section{Statistical analysis}

Data are expressed as the mean \pm SEM of at least three independent experiments for each cellular experimental group and at least five independent experiments for each animal group. We evaluated the data with Student's $t$-test. We used a one-way analysis of variance for multiple comparisons. A value of $p<0.05$ was considered significant.

\section{Results}

\section{ACR participates in the regulation of autophagy in vivo}

To investigate the potential involvement of circRNA in autophagy in the heart, we performed circRNA microarray analysis in mouse hearts with autophagy induced by $\mathrm{I} / \mathrm{R}$ injury (Fig. 1a). Among them, mmu_circRNA_006636 as indicated arrow, which we named ACR was markedly decreased after I/R injury (Fig. 1b, c). This suggests that ACR is associated with the regulation of cardiac autophagy. We further confirmed the circular nature of ARC by RNase $\mathrm{R}$ digestion (Fig. 1d). To study the effect of ACR on autophagy, we constructed a vector-based system expressing ACR as described previously [10]. The adenovirus harboring ACR resulted in an enforced expression of ACR as analyzed by northern blotting (Supplementary Figure 1A) and qRT-PCR (Supplementary Figure 1B). In vivo, delivery of ACR (Supplementary Figure 1C) attenuated the augmentation of LC3-II levels upon I/R injury (Fig. 1e). In 
A
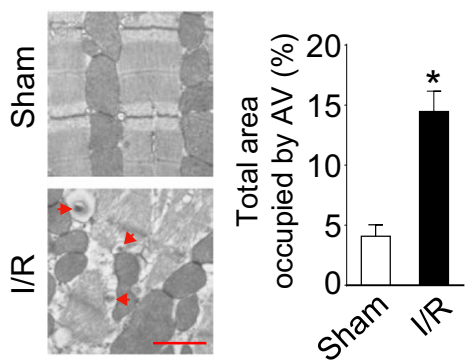

$E$

D

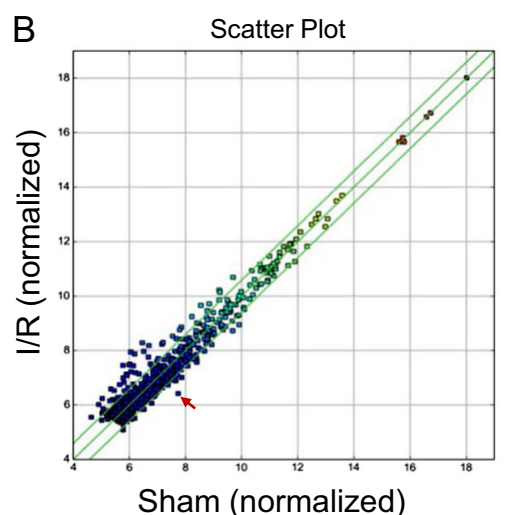

C

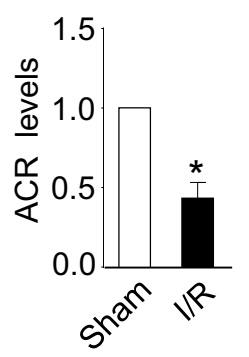

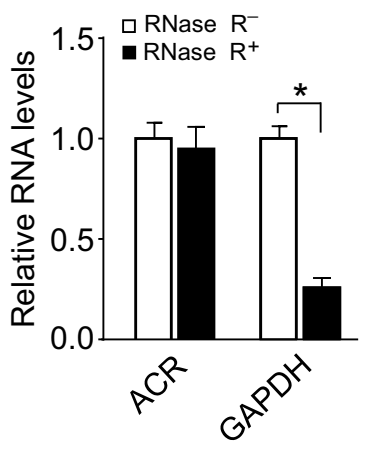

G

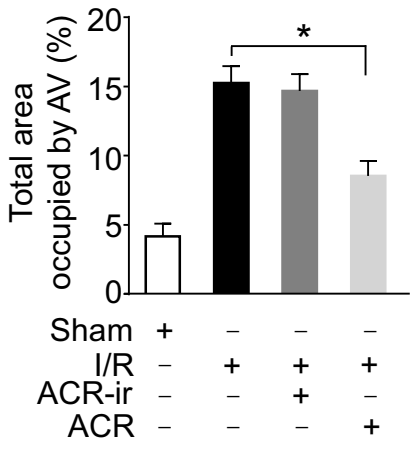

Fig. 1 ACR inhibits autophagy under the pathological condition in the heart. a Mice were subjected to $60 \mathrm{~min}$ ischemia and $3 \mathrm{~h}$ reperfusion (I/ R). Representative electron micrographs (EM) of hearts show the presence of autophagosomes (arrow). Bar $=1 \mu \mathrm{m}$. Quantification of autophagic vacuoles is shown in the right panel. $* p<0.05$. b Microarray results depicting the scatter plot of intensity of circRNA expression from Sham vs. I/R-treated mice. CircRNAs are detected by microarray. The green lines are fold-change lines. The CircRNAs above the top green line and below the bottom green line indicated more than 1.5-fold change of circRNAs between I/R and Sham. c CircRNA ACR expression in heart samples is confirmed by qRT-PCR analysis from mice subjected to $\mathrm{I} / \mathrm{R}$ or to sham treatment. $n=6$. ${ }^{*} p<0.05$ vs. sham. d Real-time PCR showing resistance of ACR to
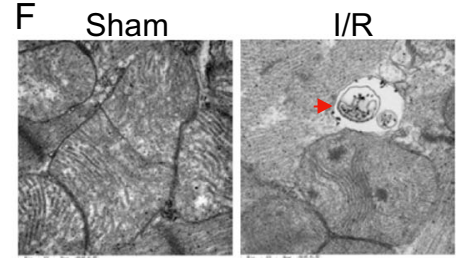

$\mathrm{I} / \mathrm{R}+\mathrm{ACR}-\mathrm{ir}$

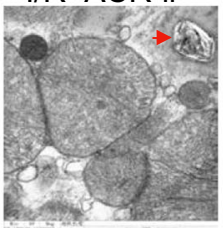

$\mathrm{l} / \mathrm{R}+\mathrm{ACR}$

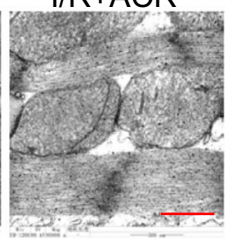

$\mathrm{H}$
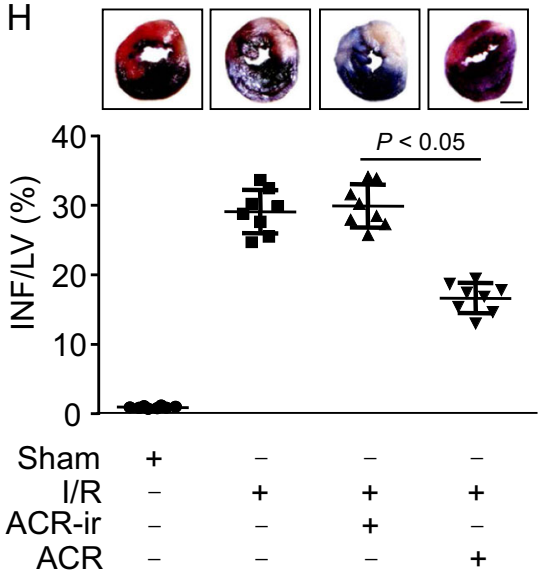

RNase R digestion. GAPDH was used as negative control. e ACR suppresses autophagy in vivo. Mice were injected with ACR or ACRir as described in Methods and then were subjected to $60 \mathrm{~min}$ ischemia and $3 \mathrm{~h}$ reperfusion (I/R). Representative immunoblot for conversion of LC3-I to LC3-II. The positions of LC3-I and LC3-II are indicated. $\mathbf{f}, \mathbf{g}$ Mice were treated as described in e. Quantification of autophagic vacuoles in the heart was analyzed by EM. $n=8$. Bar $=500 \mathrm{~nm}$. h ACR reduces myocardial infarction upon $I / R$ injury in vivo. Mice were treated as described in e. The upper panels are representative photos of midventricular myocardial slices. The low panel shows infarct sizes. Left ventricle (LV), infarct area (INF). $n=8$. Bar $=2$ mm. $* p<0.05$ 

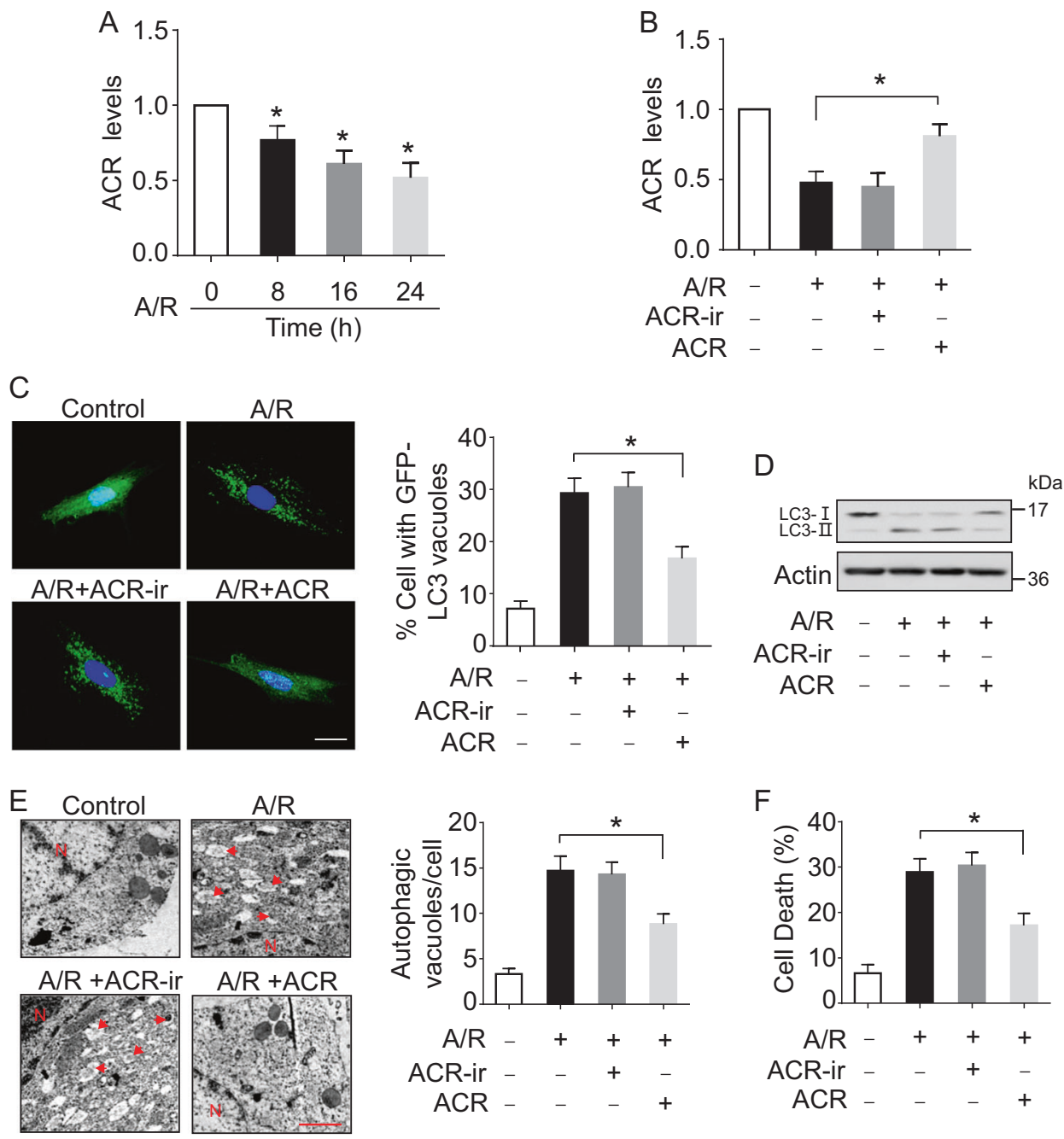

Fig. 2 ACR regulates autophagy and cell death in cardiomyocytes. a Cardiomyocytes were exposed to $\mathrm{A} / \mathrm{R}$ and ACR levels were analyzed by qRT-PCR. * $p<0.05$ vs. control. b Cardiomyocytes cells were infected with adenovirus harboring ACR or ACR-ir. Twenty-four hours after infection, cells were exposed to A/R. ACR levels are analyzed by qRT-PCR. c ACR inhibits punctate accumulations of GFP-LC3 induced by A/R. Cardiomyocytes were infected with adenovirus harboring ACR or ACR-ir, then infected with GFP-LC3. Twenty-four hours after infection, cells were exposed to A/R. Representative photos of GFP-LC3 cells were shown in the left panel and the

addition, the overexpression of ACR reduced I/R-induced extensive vacuolization in ventricular tissues, which was observed by the electron micrographic (EM) analysis (Fig. 1f and Fig. 1g). The mice treated with ACR exhibited a significant reduction of myocardial INF sizes in response to $\mathrm{I} / \mathrm{R}$ injury (Fig. 1h). In addition, I/R injury induced abnormalities in myocardial function was ameliorated in the mice treated with ACR (Supplementary Figures 2A and $2 \mathrm{~B}$ ). These data suggest that ACR participates in the regulation of autophagy signal and cell death in the heart. percentage of cells with GFP-LC3 puncta was quantified in the right panel. $\mathrm{Bar}=20 \mu \mathrm{m}$. d Representative immunoblot for conversion of LC3-I to LC3-II. Cells were treated as described in $\mathbf{b}$. The positions of LC3-I and LC3-II are indicated. e ACR reduces autophagic vacuoles. Cardiomyocytes were treated as described in b. Representative EM images were shown in the left panel. Bar $=2 \mu \mathrm{M}$. The arrows depict autophagosomes and the nucleus is denoted by N. Quantification of autophagic vacuoles was shown in the right panel. f ACR attenuates A/ R-induced cell death. Cells were treated as described in e. Quantification of cell death was shown

\section{ACR inhibits autophagy and cell death in cardiomyocytes}

Next, we examined the role of ACR in autophagy in cardiomyocytes. The accumulation of GFP-LC3 puncta is an indicator of increased autophagosomes and autophagy. We observed that A/R increased GFP-LC3 punctuate structures (Supplementary Figure 2C) and caused a decreased expression of ACR in cardiomyocytes (Fig. 2a). The enforced expression of ACR (Fig. 2b) significantly reduced the punctate accumulations as indicated by reduced GFP- 
Fig. 3 ACR participates in the regulation of Pink1 expression. a Knockdown of ACR reduces the ACR expression.

Cardiomyocytes were infected with adenoviral ACR-siRNA or ACR-sc. ACR levels were analyzed by qRT-PCR

b Volcano plot of the microarray results. Cardiomyocytes were treated as described in a.

Volcano plot of the fold changes of the genes ( $\log 2$ scale) vs. the $P$-values of the genes ($\log 10$ scale). The dots in the plot represent an average of three microarray slides results. c Autophagy-related genes Irgm1 and Pink1 from microarray are confirmed by qRT-PCR. d, e Cardiomyocytes were infected with adenoviral ACR-siRNA or ACR-sc. Irgm1 levels (d) and Pink1 levels (e) were analyzed by immunoblot. f ACR induces the increase of Pink1 expression levels. Cardiomyocytes were infected with adenoviral ACR-ir or ACR. Pink1 expression was analyzed by qRT-PCR (upper panel) and immunoblot (low panel). g, h ACR has no effect on Irgm1 expression.

Cardiomyocytes were infected with adenoviral ACR-ir or ACR Irgm1 expression was analyzed by qRT-PCR (g) and immunoblot (h)
A

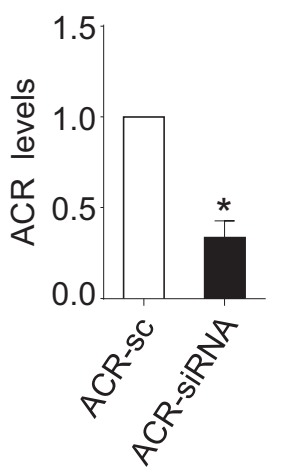

C

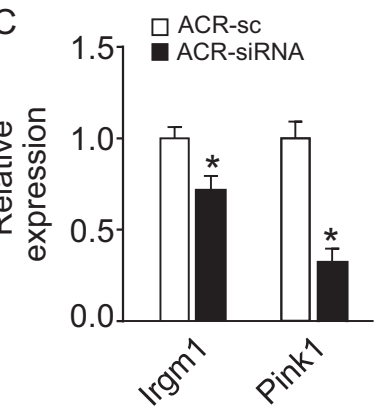

$\mathrm{F}$

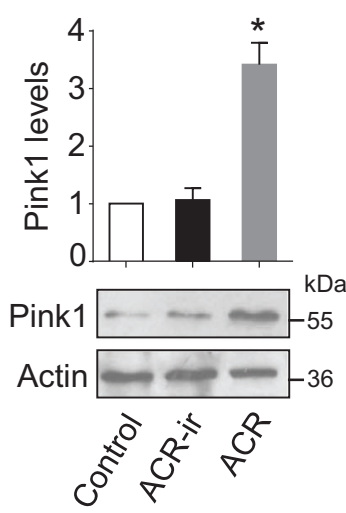

B volcano plot (ACR-siRNA vs ACR-sc)

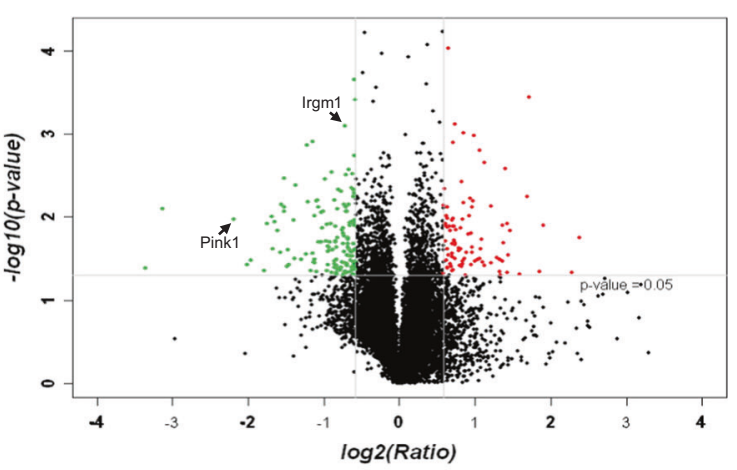

D

E
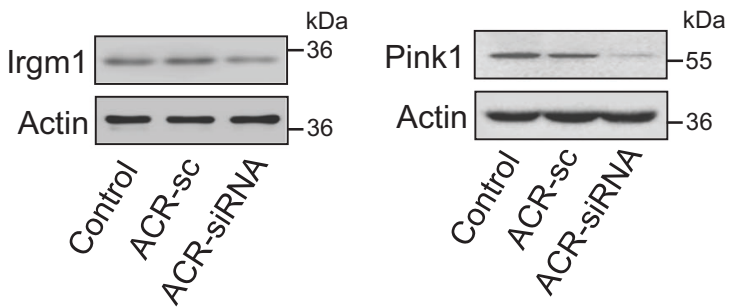

G

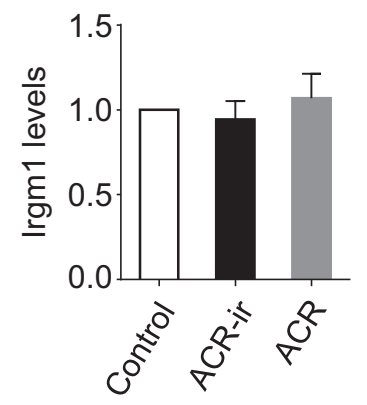

$\mathrm{H}$

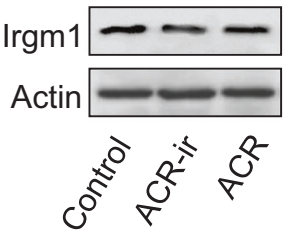

LC3 (Fig. 2c) and LC3-II expression in A/R-treated cells (Fig. 2d). The EM analysis showed that ACR reduced autophagic vesicles in cardiomyocytes with A/R (Fig. 2e) and it also attenuated cell death induced by A/R (Fig. 2f). Mitophagy is a specific and selective form of autophagy and thus we also investigated the effect of ACR on mitophagy. The mitophagy was upregulated after I/R injury (Supplementary Figure 3A) and A/R (Supplementary Figures 3B and $3 \mathrm{C}$ ), and overexpression of $\mathrm{ACR}$ attenuated $\mathrm{A} / \mathrm{R}$ induced mitophagy as evidenced by decreased colocalization of LC3 with mitochondria (Supplementary Figures 3B and 3C), whereas knockdown of ACR with siRNA remarkably upregulated mitophagy activity (Supplementary Figures 3D and 3E). Taken together, these results suggest that ACR inhibits A/R-induced autophagy, mitophagy, and cell death in cardiomyocytes.

\section{ACR regulates Pink1 expression}

To further investigate the function of ACR, we searched its downstream targets by performing the transcriptome microarray. We found that the expression of a variety of genes were altered upon ACR knockdown (Fig. 3a and Supplementary Figure 4A). Gene Ontology functional enrichment analysis was performed for differentially expressed genes (Supplementary Figure 4B). The functional enrichment analysis showed that the differentially expressed genes could be involved in biological processes, such as cellular response to starvation, positive regulation of fibroblast growth factor receptor signaling pathway, and regulation of autophagy. Among those genes, we focused on autophagy-related genes Irgm1 and Pink1, which were dramatically altered in ACR knockdown cardiomyocytes (Fig. 3b). 
We analyzed the expression of Irgm 1 and Pink1 by quantitative PCR (qPCR) and found that Pink1 was dramatically decreased in ACR knockdown cardiomyocytes, whereas it had little effect on the expression of Irgm1 (Fig. 3c-e). In contrast, ACR-overexpressing cells showed a marked elevation in the level of Pink1 expression (Fig. 3f) compared with Irgm1 expression (Fig. $3 g$, h). These results indicate that ACR is able to regulate the expression of Pink1, and Pink1 may act as a potential downstream target in ACR-mediated autophagy process.

\section{ACR inhibits DNA methylation of Pink1 by binding to DNMT3B}

We sought to gain further mechanistic insight into the regulatory role of ACR in Pink1 expression. DNA methylation has been demonstrated to control the local gene expression. The presence of a strong $\mathrm{CpG}$ island at the Pink1-associated promoter (Supplementary Figure 5A) suggests that Pink1 may be under control by DNA methylation and ACR likely modulates its expression through regulating the promoter methylation. To test whether that Pink1 transcription can be regulated by DNA methylation, we treated cardiomyocytes with methylation inhibitor 5'Aza-2'-deoxycytidine (5-Aza). As expected, 5-Aza treatment induced an increase in Pink1 expression (Supplementary Figure 5B). We performed bisulfite sequence analysis to explore whether ACR regulates Pink1 expression through promoter DNA methylation. The analysis of CpG methylation by bisulfite sequencing demonstrated that ACR knockdown in cardiomyocytes increased the degree of methylation of the Pink1 promoter (Fig. 4a).

The regulation of ACR on Pink1 promoter methylation prompted us to speculate whether ACR could recruit or associate the members of the DNA methyltransferases (DNMTs) family. To test whether ACR physically associates with DNMTs, we performed RNA immunoprecipitation (RIP) using antibodies against maintenance enzyme Dnmt1 and de novo enzymes Dnmt3A or Dnmt3B. We observed ACR enrichment in DNMT3B-RNA precipitates, whereas Dnmt1 RNAs and Dnmt3A RNAs were not retrieved, which indicates a physical interaction between ACR and Dnmt3B (Fig. 4b). Next, we performed RNA pull-down assay using biotinylated ACR probe and found that a substantial amount of Dnmt3B was pulled down with ACR probe (Fig. 4c). We further explored the mechanism by which ACR inhibits Pink1 promoter methylation. RNA pull-down followed by western blot analysis showed that ACR knockdown caused a significant decrease in the binding of Dnmt3B (Fig. 4d). The results of quantitative ChIP (qChIP) experiments showed that ACR knockdown enhanced the binding of DNMT3B to the $\mathrm{CpG}$ region of Pink1 promoter (Fig. 4e), which indicates that ACR blocks the association of Dnmt3B with Pink1 promoter. In addition, we found that Dnmt3B knockdown (Supplementary Figure 5C) increased PINK1 expression (Fig. 4f), whereas enforced expression of ACR counteracted the inhibitory effect of Dnmt3B on Pink1 expression (Fig. 4g). Furthermore, the increased binding between Dnmt3B and PINK1 promoter was observed in response to $A / R$ treatment (Fig. 4h) or I/R injury (Fig. 4i). Collectively, these data suggest that ACR binds to Dnmt3B in vivo and, therefore, block Dnmt3B-mediated DNA methylation of Pink1.

\section{Pink1 regulates the autophagy in cardiomyocytes}

Next, we tested whether Pink1 plays a functional role in autophagy. Enforced expression of Pink1 (Fig. 5a and Supplementary Figure 5D) reduced GFP-LC3-II punctate accumulations (Fig. 5b) in A/R-treated cells (Supplementary Figure 5E). Pink1 overexpression also attenuated autophagic vesicles (Fig. 5c) upon A/R treatment. Pink1 reduced the levels of LC3-II (Fig. 5d, upper panel) and cell death (Fig. 5d, low panel) in A/R-treated cells. These results suggest that Pink1 mediates the autophagy and cell death in cardiomyocytes.

\section{Pink1 regulates the autophagic signal in vivo}

To confirm this phenomenon in vivo condition, we generated transgenic mice with cardiac-specific expression of Pink1 by constructing Pink1 sequence under the control of $\alpha$-myosin heavy chain promoter (Fig. 6a). These mice developed normally to adulthood without significant alterations in terms of phenotype and cardiac function under physiological condition. For this study, we generated five independent transgenic mice founder lines (Line\#1 to Line\#5). All the lines stably expressed Pink1 in adult hearts in which the expression of Pink1 was about two to fivefold higher than that of in WT hearts (Supplementary Figure 5F). For our study, we randomly chose Pink1 transgenic mice generated from Line\#1, which showed about fourfold increase of Pink1 in adult mouse hearts, and these mice exhibited an increase in the level of Pink1 compared with WT group following I/R injury (Fig. 6b). Pink1 transgenic mice showed a reduction in autophagy, which was revealed by EM analysis of ventricular tissue (Fig. 6c, d). The I/R injury induced increase of myocardial INF was significantly reduced (Fig. 6e) and myocardial function was significantly improved (Fig. 6f) in Pink1 transgenic mice. These data suggest that Pink1 contributes to the regulation of autophagy signal and cell death in the heart.

\section{Pink1 targets and phosphorylates FAM65B}

To investigate the underlying mechanism by which Pink1 regulates autophagy, we made attempt to identify the downstream target of Pink1. Pink1 has a highly conserved 
A

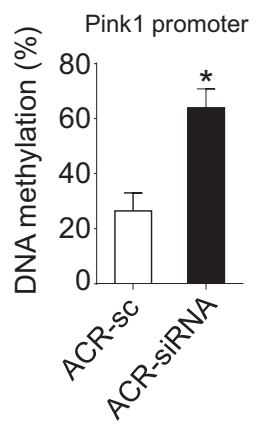

D
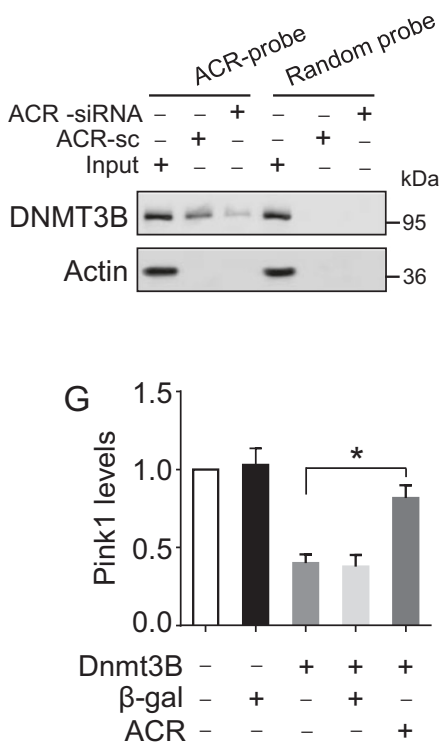

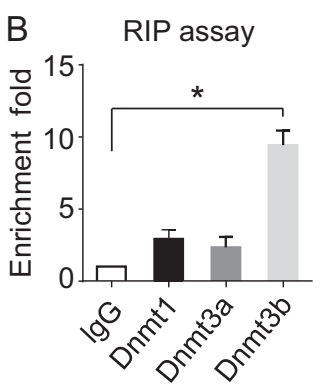

$\mathrm{E}$

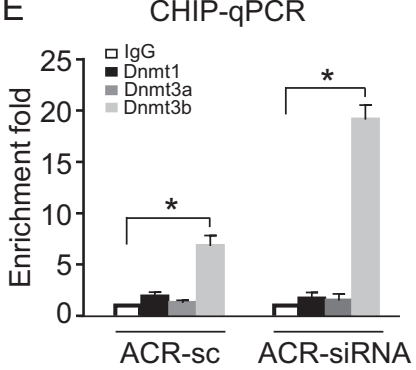

$\mathrm{H}$

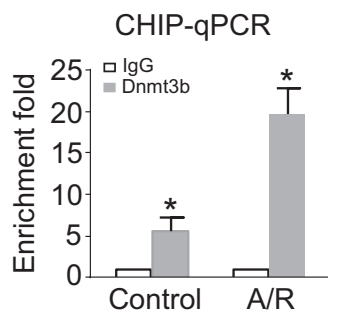

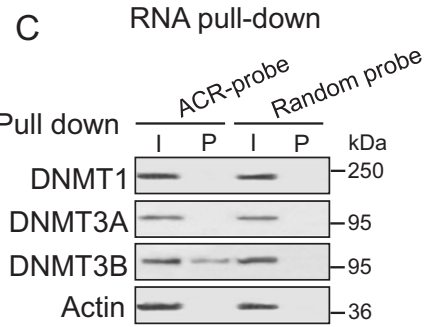

$\mathrm{F}$

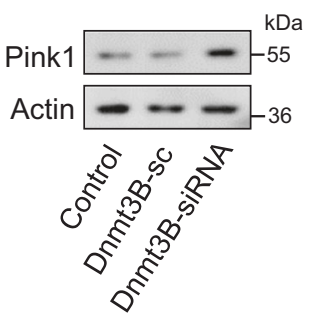

I

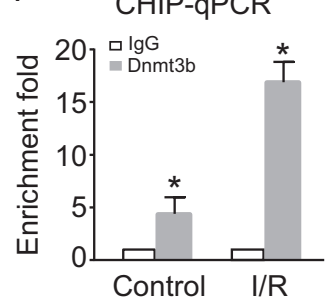

Fig. 4 ACR interacts with DNMT3B to block Pink1 promoter methylation. a Bisulfite sequencing analysis of the Pink1 promoter. Cardiomyocytes were infected with adenoviral ACR-siRNA or ACRsc. The degree of methylation was increased in ACR knockdown cardiomyocytes as showed by percentage of the increased methylated cytosines. $n=6$. b RNA immunoprecipitation (RIP) was performed using antibodies against DNMT1, DNMT3A, or DNMT3B in cardiomyocytes. ACR bound to DNMT3B as revealed by RIP assay. $n=3$. c RNA pull-down assay: cardiomyocytes were collected and mixed with biotinylated ACR probe or random probe-coated magnetic bead to perform biotin-based pull-down assay. DNMTs levels were analyzed by western blotting. $n=4$. d Knockdown of ACR decreases its interaction with DNMT3B. Cardiomyocytes were infected with adenovirus ACR-siRNA or ACR-sc. Forty-eight hours after infection, RNA pull-down assay was performed and DNMT3B protein was

protein kinase domain [44] and its kinase activity is poorly described. FAM65B can promote myogenic cell differentiation. It is highly conserved among species [45] and it can be phosphorylated [46]. Co-immunoprecipitation result showed that Pink1 could bind to FAM65B (Fig. 7a). The protein sequence of FAM65B was screened by MS and we identified that S46 was phosphorylated (Fig. 7b). S46 is conserved among mouse, rat, and human (Fig. 7c). To confirm the phosphorylation site at S46, we raised antibodies against FAM65B phosphorylation at sites S46, detected by western blotting. e ACR knockdown enhances the enrichment of DNMT3B on Pink1 promoter. Cardiomyocytes were infected with adenovirus ACR-siRNA or ACR-sc. Forty-eight hours after infection, ChIP-qPCR was performed. $\mathbf{f}$ Cardiomyocytes were infected with adenoviral Dnmt3B-sc or Dnmt3B-siRNA. Pink1 expression was analyzed by immunoblotting. $\mathbf{g}$ ACR counteracts the inhibitory effect of DNMT3B on Pink1 expression. Cardiomyocytes are infected with adenoviral DNMT3B, $\beta$-gal, or ACR. Pink1 levels are analyzed by qRT-PCR. $\mathbf{h} \mathrm{A} / \mathrm{R}$ treatment enhances the enrichment of DNMT3B on Pink1 promoter. Cardiomyocytes were exposed to A/ R. Forty-eight hours after treatment, ChIP-qPCR was performed. i I/R injury enhances the enrichment of DNMT3B on Pink1 promoter. Mice were subjected to $\mathrm{I} / \mathrm{R}$ as described in Methods. ChIP-qPCR was performed

T119, and S558, and the latter two used as a control. Only S46 is phosphorylated (Fig. 7d). We generated two mutants: one converts S46 to A46 and another one converts S44 to A44, which served as a control. Using transcription- and translation-coupled rabbit raticulocytes, we found that the S46A mutant could not be phosphorylated (Fig. 7e). The knockdown of Pink1 (Fig. 7f) led to a decrease in S46 phosphorylation, whereas the total expression level of FAM65B remained unchanged (Fig. $7 \mathrm{~g}$ ). We also generated the Pink1 kinase dead mutant (K219A/D362A/D384A) to 

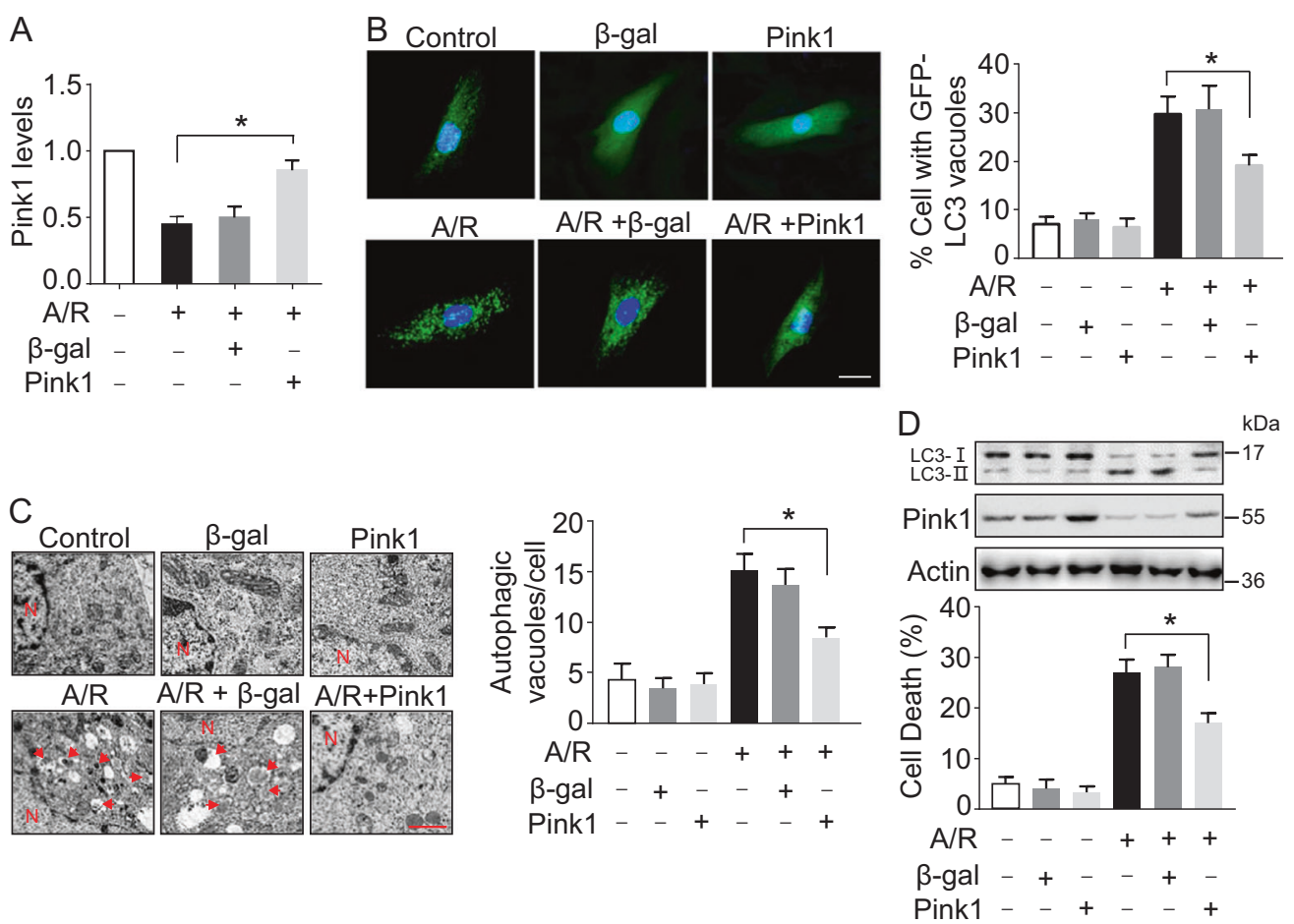

Fig. 5 Pink1 suppresses autophagy and cell death in cardiomyocytes. a Cardiomyocytes cells were infected with adenovirus harboring Pink1 or $\beta$-gal. Twenty-four hours after infection, cells were exposed to A/R. Pink1 levels are analyzed by qRT-PCR. b Pink1 inhibits punctate accumulations of GFP-LC3 induced by A/R. Cardiomyocytes were infected with adenovirus harboring Pink1 or $\beta$-gal, then infected with GFP-LC3. Twenty-four hours after infection, cells were exposed to A/R. Representative photos of GFP-LC3 cells were shown in the left panel, and the percentage of cells with GFP-LC3 puncta was

study the kinase activity of Pink1 [21, 47]. The kinase dead mutation of Pink1 resulted in reduced S46 phosphorylation (Fig. 7h). These data indicate that FAM65B can be phosphorylated at S46 by Pink1.

\section{ACR exerts its autophagic effect through Pink1 and FAM65B}

Then, we investigated whether Pink-dependent phosphorylation of FAM65B has a role in autophagy. The phosphorylated FAM65B but not total FAM65B level was decreased upon $A / R$ treatment (Fig. 8a), and this decrease was rescued by the expression of Pink 1 wt but not KDD mutant (Supplementary Figure 6A). The overexpression of FAM65B mutant with the conversion of residue S46 into an alanine (FAM65B-46A) did not increase FAM65B phosphorylation level when compared with its phosphorylation level in WT FAM65B (FAM65B-wt) overexpressed cells (Fig. 8b). These results suggest that phosphorylation of Serine 46 may account for the activity of FAM65B. In addition, FAM65B-wt but not FAM65B-46A significantly quantified in the right panel. $\mathrm{Bar}=20 \mu \mathrm{m}$. c Cardiomyocytes were treated as described in a. Representative EM images were shown in the left panel. Bar $=2 \mu \mathrm{M}$. The arrows depict autophagosomes and the nucleus is denoted by N. Quantification of autophagic vacuoles was shown in the right panel. d Cells were treated as described in a. Representative immunoblot for conversion of LC3-I to LC3-II and Pink1 expression were shown in the upper panel. Quantification of cell death was shown in the low panel

attenuated A/R-induced cardiomyocytes autophagy (Fig. 8c and Fig. 8d) and cell death (Fig. 8e).

Next, we tested whether phosphorylated FAM65B is involved in ACR-mediated regulation of autophagy. We detected the effect of ACR on the phosphorylation of FAM65B. Knockdown of ACR significantly reduced the phosphorylation of FAM65B (Fig. 8f), which was rescued by the expression of Pink1 wt but not KDD mutant (Supplementary Figure 6B). Overexpression of ACR increased the level of phosphorylated FAM65B (Fig. 8g) and knockdown of Pink1 attenuated ACR-induced increase of phosphorylated FAM65B (Fig. 8h). In addition, the overexpression of ACR inhibited A/R-induced decrease of phosphorylated FAM65B level and the increase of autophagy, whereas the knockdown of Pink1 counteracted the ACR-induced increase of FAM65B phosphorylation (Fig. 8i) and decrease of autophagy (Fig. 8j). Together, these data suggest that ACR exerts its inhibitory effect on autophagy and cell death through Pink1-dependent phosphorylation of FAM65B. 
Fig. 6 Pink1 suppresses autophagy and myocardial infarction in vivo. a qRT-PCR analyses the expression of Pink1 in different tissues isolated from Pink1 transgenic mice (Tg) and wild-type mice (WT). b WT and Pink1 transgenic mice were subjected to $\mathrm{I} / \mathrm{R}$ as described in Methods. Pink1 levels were detected by immunoblot. c, d Autophagy was reduced in Pink1 transgenic mice upon I/R. Pink1 transgenic mice (Pink1-

$\mathrm{Tg}$ ) or wild-type mice (WT) were subjected to $60 \mathrm{~min}$ ischemia and $3 \mathrm{~h}$ reperfusion (I/R). The representative electron micrographs of hearts were shown. e Pink1 transgenic mice exhibit reduced myocardial infarction upon I/R. Pink1 transgenic mice (Tg) and wildtype mice (WT) were exposed to I/R. The upper panels are representative photos of midventricular myocardial slices. The low panel shows infarct sizes. Left ventricle (LV), infarct area (INF). $n=6$. Bar $=$ $2 \mathrm{~mm} . P<0.05$. f WT and Pink1 transgenic mice were subjected to $60 \mathrm{~min}$ of ischemia followed by 1 week of reperfusion (I/R). Transthoracic echocardiographic analysis was performed. FS, fractional shortening of left ventricular diameter. ${ }^{*} p<0.05$

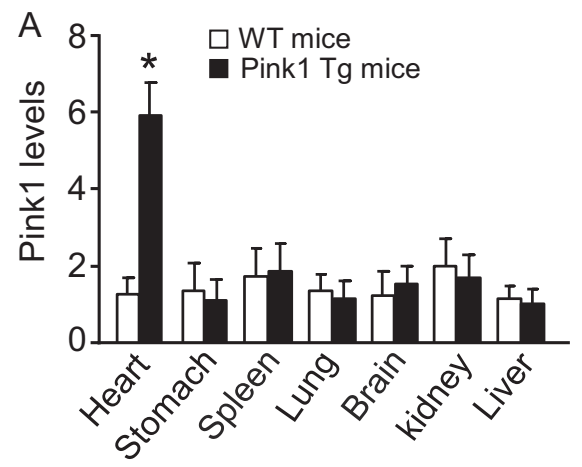

C

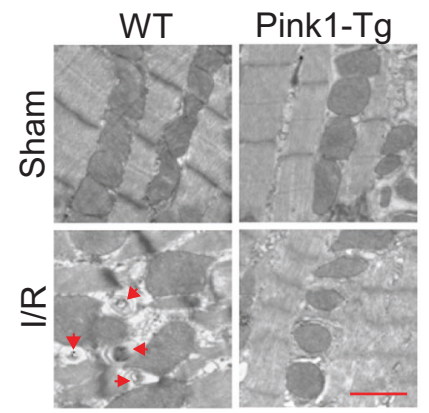

E
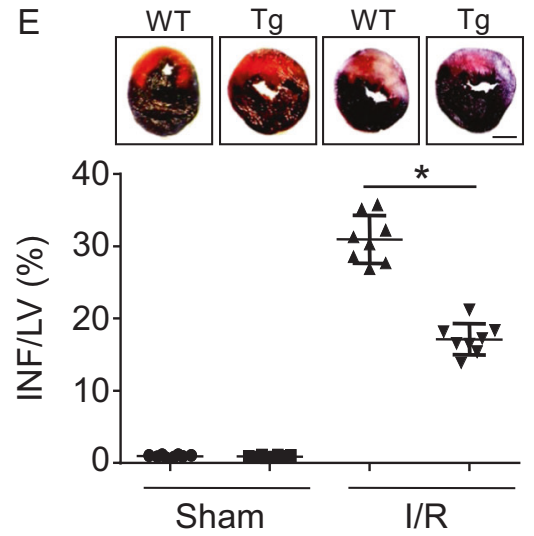

B

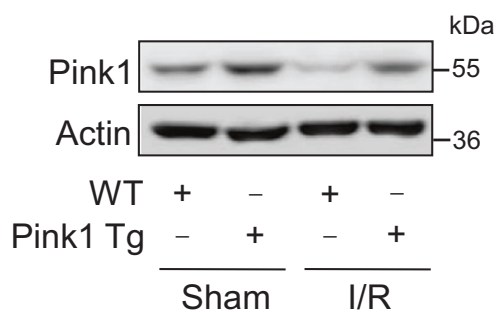

D

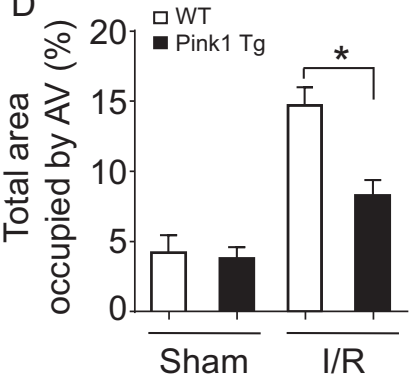

F

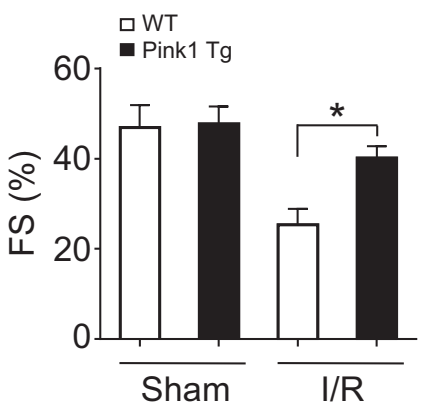

\section{Discussion}

Hitherto, it is generally considered that maintenance of autophagy below physiological level is usually beneficial for cell survival, while uncontrolled excessive induction of autophagy is detrimental and it inevitably leads to cell death. Accumulating evidences suggest that the inhibition of autophagic cell death protects cardiomyocytes during ischemia/reperfusion [48]. It is well known that autophagy is the most prominent mechanism that results in the death of cardiomyocytes in end-stage heart failure [49]. In addition, the inhibition of autophagy ameliorates the pathological remodeling of the LV [50]. In accordance with these studies, our current findings demonstrate that $\mathrm{A} / \mathrm{R}$ and $\mathrm{I} / \mathrm{R}$ injury induce autophagy and cardiomyocytes death. The inhibition of autophagy attenuates A/R-induced cardiomyocytes cell death and I/R-induced myocardial INF.
Currently, the beneficial and detrimental features of autophagy remain obscure and more detailed investigations are needed to further clarify the role of autophagy during I/Rinduced myocardial injury.

In the present study, we identified a circRNA-mediated regulation of autophagy pathway involving Pink1 and FAM65B. Emerging evidences have shown that circRNAs have important roles in many biological processes including the regulation of atherosclerosis [51], cell growth [52], and proliferation and invasion of cancer cells [53, 54]. However, there is no report available to depict the functional role of circRNAs on autophagy. Here we, for the first time, demonstrate that a new circRNA (ACR) is able to mediate autophagy in cardiomyocytes. ACR inhibits autophagy and cell death in cardiomyocytes and it protects heart from I/R injury by attenuating I/R-induced increase of myocardial INF sizes. Our results provide new insights for 
A

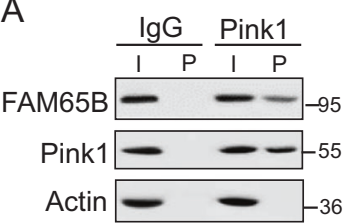

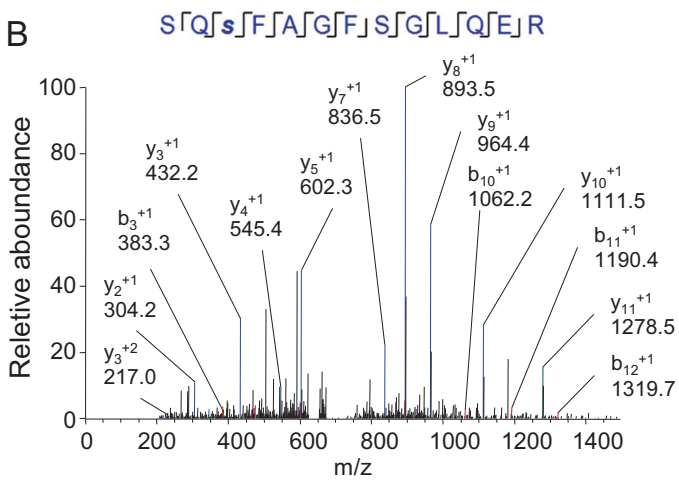

C
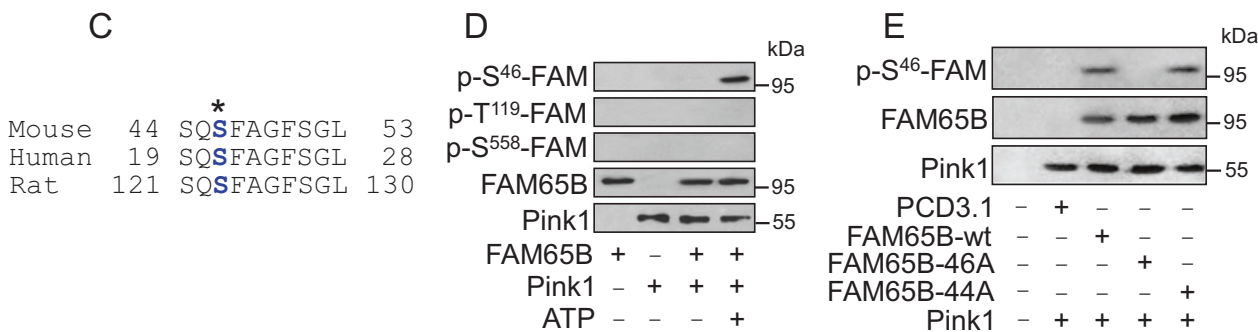

$\mathrm{F}$

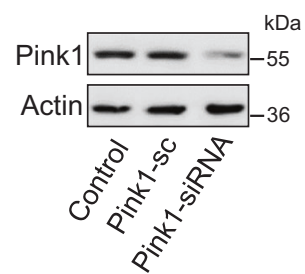

G

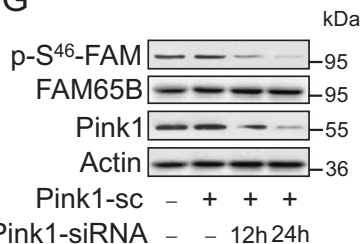

$\mathrm{H}$

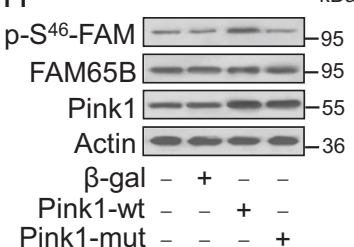

Fig. 7 FAM65B is phosphorylated at $\mathrm{S} 46$ by Pink1. a Pink1 is associated with FAM65B. The lysates of cardiomyocytes were immunoprecipitated with the anti-Pink1 antibody or anti-IgG antibody. The immunoprecipitates were analyzed by immunoblot using the antiFAM65B antibody and Pink1 antibody. b FAM65B phosphorylation detected by mass spectrometry. 46SQSpFAGFSGLQER (Sp-phosphoserine residue) with $\mathrm{m} / \mathrm{z}=747.38483 \mathrm{Da}, \mathrm{MH}+=1493.76238$ $\mathrm{Da}$. The mass of the $\mathrm{y}$-ions corresponds to the nonphosphorylated Cterminal part, whereas the b-ions confirm the phosphorylation site. c The potential phosphopeptide identified in FAM65B is highly conserved between species. The identified phosphorylation site is marked (*). d In vitro phosphorylation of FAM65B by Pink1. Purified FAM65B $(0.7 \mu \mathrm{g})$ was tagged by Histidine. The cMyc-tagged Pink1 was expressed in HEK293T cells and immunoprecipitated by the anticMyc antibody. The immunoprecipitated Pink1-cMyc was incubated with recombinant-purified FAM65B-His protein in kinase buffer w/o ATP $(400 \mu \mathrm{M})$. The levels of FAM65B phosphorylation were analyzed by immunoblot using the antibody against phospho-S46, phospho-

understanding the function of circRNAs in cardiomyocyte autophagy and offer a new strategy to treat myocardial INF and heart failure.

Previous studies have shown that circRNAs can regulate gene exrpression through several molecular mechanisms. For example, circRNAs act as miRNA sponge and influence the expression of miRNA target genes $[6,10,55]$. CircANRIL modulates ribosomal RNA
T119, and phospho-S558. Total FAM65B and Pink1 levels were also analyzed by immunoblotting. e FAM65B is phosphorylated at S46 in the in vitro translation reticulocyte lysate system. Pink1 was produced in the in vitro translation reticulocyte lysate system together with FAM65B and its mutants. pcDNA3.1 is the control empty plasmid. The products were analyzed by immunoblot using the antibody against phospho-S46 and Pink1. f Knockdown of Pink1 reduces the Pink1 expression. Cardiomyocytes were infected with adenoviral Pink1siRNA or Pink1-sc. Pink1 levels were analyzed by immunoblot. g Knockdown of Pink1 reduces FAM65B phosphorylation at S46. Cardiomyocytes were treated with Pink1-siRNA constructs or Pink1sc, and harvested for immunoblot. h Pink1 kinase activity is essential for FAM65B phosphorylation. Cardiomyocytes were infected with adenovirus harboring Pink1-wt, Pink1-mut, or $\beta$-gal, and then the levels of FAM65B phosphorylation and total FAM65B were analyzed by immunoblot. Pink1-mut(KDD) is a triple mutant without kinase activity (K219A/D362A/D384A)

maturation by circRNA-protein interaction [51]. EIciRNAs localizes in the nucleus in which it interacts with U1 snRNP and promotes transcription of their parental genes [56]. In our current study, we for the first time demonstrate that circRNA regulates gene expression through affecting the DNA methylation of its downstream gene. Our results show that circRNA ACR activates Pink1 expression through directly binding to 
A

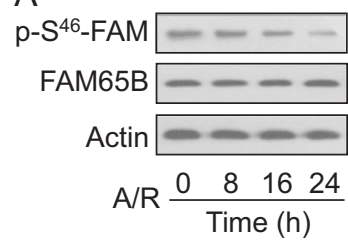

$B$

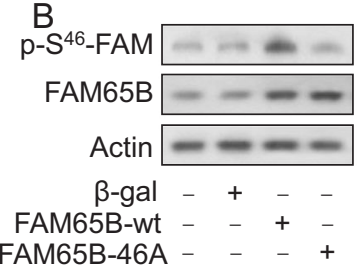

FAM65B ----

Actin $=--m$

$\beta$-gal -+-

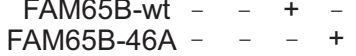

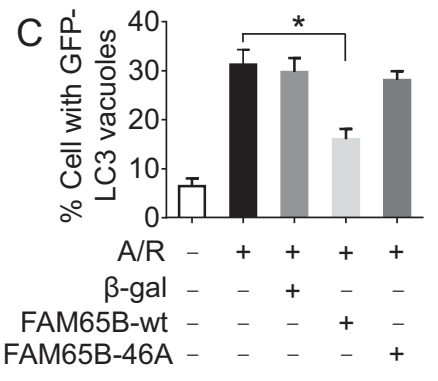

$\mathrm{F}$

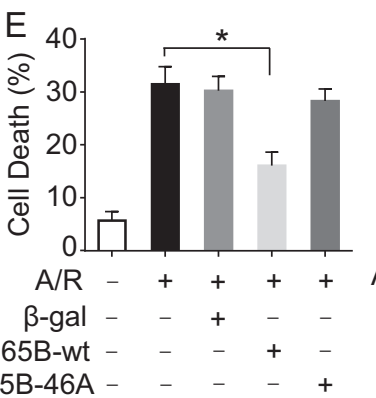

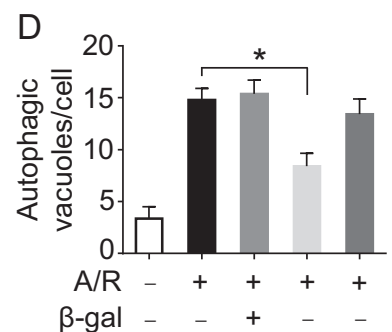

FAM65B-wt FAM65B-46A -
FAM65B-wt

G

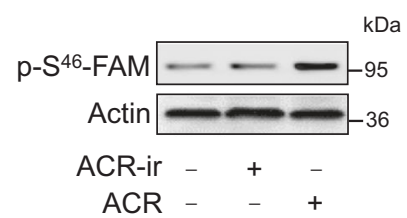

I

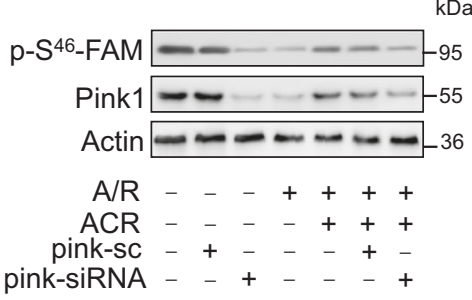

Fig. 8 ACR exerts its autophagic effect through Pink1 and phosphorylated FAM65B. a Phosphorylated FAM65B level was decreased upon $A / R$ treatment. Levels of phosphorylated FAM65B and total FAM65B were detected by immunoblot. b Cardiomyocytes were infected with adenovirus harboring FAM65B-wt, FAM65B-46A, or $\beta$ gal, and then the levels of FAM65B phosphorylation and total FAM65B were analyzed by immunoblot. c FAM65B-wt attenuates punctate accumulations of GFP-LC3 induced by A/R. Cardiomyocytes were infected with adenovirus harboring FAM65B-wt, FAM65B-46A, or $\beta$-gal, then infected with GFP-LC3. Twenty-four hours after infection, cells were exposed to $\mathrm{A} / \mathrm{R}$. The percentage of cells with GFP-LC3 puncta was quantified. d Cardiomyocytes were infected with adenovirus harboring FAM65B-wt, FAM65B-46A, or $\beta$-gal, then were exposed to A/R. Quantification of autophagic vacuoles was shown. e Cells were treated as described in $\mathbf{d}$. Quantification of cell death was

Dnmt3B and this interaction blocks Dnmt3B-mediated DNA methylation of Pink1 promoter. This study extends our knowledge about the mechanisms of

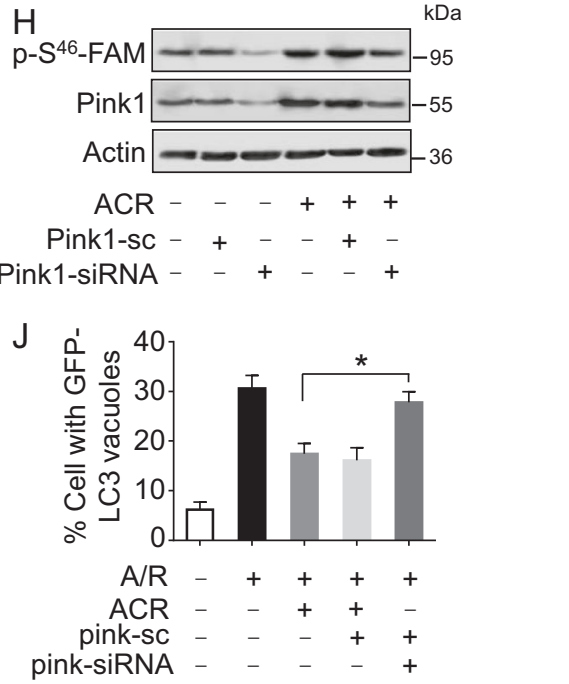

shown. f Knockdown of ACR reduces the expression levels of phosphorylated FAM65B. Cardiomyocytes were infected with adenoviral ACR-siRNA or ACR-sc. Forty-eight hours after infection, phosphorylated FAM65B levels were analyzed by immunoblotting. g Enforced expression of ACR induces an increase in phosphorylated FAM65B levels. Cardiomyocytes are infected with adenoviral ACR or ACR-ir, and phosphorylated FAM65B levels are analyzed by immunoblotting. $\mathbf{h}$ Knockdown of Pink1 counteracts the effect of ACR on phosphorylated FAM65B expression. Cardiomyocytes are infected with adenoviral ACR, Pink1-siRNA or Pink1-sc. Phosphorylated FAM65B levels were analyzed by immunoblot. i, j Cardiomyocytes are infected with adenoviral ACR, Pink1-siRNA, or Pink1-sc. Twentyfour hours after infection, cells were exposed to A/R. Phosphorylated FAM65B levels were analyzed by immunoblotting (i). The percentage of cells with GFP-LC3 puncta was quantified (j)

circRNA-protein interaction and the involvement of circRNAs in the regulation of DNA methylation. This newly identified regulatory mechanisms of circRNAs 
will provide a better understanding of circRNA-mediated gene regulation.

Numerous studies show that PINK1 is associated with the induction of mitophagy and clearance of damaged mitochondria through autophagy. In recent years, emerging evidences indicate that PINK1 has differential impact on mitophagy/autophagy depending on the stimulus and cellular environment. Interestingly, PINK1- and/or Parkin (a direct downstream of PINK1)-dependent mitophagy differs between neural cells and non-neural cells [57]. A study by Rakovic found that the endogenous PINK1 can attenuate mitophagy by ubiquitin proteosome system-dependent degradation of Parkin, which results in decreased level of Parkin and subsequently attenuates mitophagy [57]. Another research group also found that the overexpression (transient and stable) of PINK1 suppresses the mitophagic response and autophagy caused by toxins or other stresses in human neuroblast cells (SY-SY5Y cells) [58]. Likewise, the silencing of PINK1 induces activation of mitochondrial degradation process and promotes autophagy in mouse embryonic fibroblasts [59] and dopaminergic MN9D cells [60]. Qi et al. [61] also confirmed this phenomenon in dopaminergic MN9D cells in which knockdown of PINK1 promotes autophagosome formation and autophagic process. In fact, the native PINK1 has the capability to inhibit basal and toxic substances-inducible apoptotic cell death in neural cells by preserving mitochondrial integrity and attenuating the release of pro-apoptotic factors such as cytochrome C [62].

There are several mechanisms proposed for the PINK1mediated suppression of mitophagy. A recent study indicates that the cytoplasmic level of cleaved products of PINK1 has important role in suppressing mitophagy. PINK152 is a major cleaved product of PINK1 that directly interacts with Parkin and it interferes Parkin translocation to mitochondria, which results in decline in the level of mitophagy [63]. In addition, PINK1 tightly controls mitochondrial integrity and generation of reactive oxygen species and thereby it stabilizes mitochondrial network, prevents the loss of mitochondrial membrane potential, suppresses the mitochondrial fragmentation, and ultimately attenuates mitophagy/autophagy $[58,60]$. In fact, the endogenous PINK1/Parkin-dependent mitophagy occurs at much lower level under normal physiological conditions. However, any impairment in PINK1 function/expression accelerates mitophagy due to increased level of Parkin. In addition, if the loss of mitochondrial membrane potential and mitochondrial damage is excessive beyond the limit, PINK1 guides the translocation of Parkin to the mitochondrion and promotes mitophagy [57] as observed in the majority of studies. This study clearly indicates the dual state of PINK1 in the induction of mitophagy. Despite the mechanisms of PINK1dependent inhibition and activation of autophagy is currently unclear, the available reports indicate that the distinct functions of PINK1 in mitophagy/autophagy rely on the cellular environment, as well as the stability, different subcellular pools, and downstream partners of PINK1. In this context, we found that PINK1 acts as a negative regulator of autophagy in cardiomyocytes. In addition, we found that FAM65B acts as a downstream of PINK1, which activates FAM65B by phosphorylation at serine 46 to suppress the autophagy in cardiomyocytes. Thus, our finding not only reveals the new mechanism of PINK1 function, but also indicates that PINK1 could have many non-classical downstream effectors, which might be responsible for the differential functions of PINK1. Further detailed studies are warranted to address this phenomenon.

Until now, there is no study available to show the role of FAM65B in cardiomyocytes as well as its role in autophagy. Our work for the first time demonstrates the function role of FAM65B in autophagy in cardiomyocytes. In addition, we also identified that FAM65B is a downstream target of Pink1 and it is phosphorylated at serine 46 by Pink1. Phosphorylated Fam65B can interact with HDAC6 and 14.3.3 protein, and has important cellular function [33, 34]. Our data show that the phosphorylated FAM65B has capability to suppress autophagy and cell death in the heart. Fam65B can also be phosphorylated by other factors, such as chemokine and chemokine stimulates Fam65B phosphorylation in $\mathrm{T}$ lymphocytes [64]. In our study, the overexpression of Pink1 only causes partial rescue of Fam65B phosphorylation during A/R, which suggests that other factors might also participate in regulating Fam65B phosphorylation during autophagy. It would be interesting to investigate whether chemokine and/or other regulators are involved in the A/R-induced regulation of autophagy in the future. Furthermore, further studies are warranted to unveil whether there is a relationship between FAM65B and expression and/or activities of autophagy components, such as Beclin 1, Atg5, Atg7, etc.

\section{Acknowledgements}

Funding This work was supported by CAMS Innovation Fund for Medical Sciences (2016-12M-1-006), National Natural Science Foundation of China (81522005, 81770275, 81828002, 81470522, and 81500222). Taishan Scholar Program of Shandong Province and Natural Science Foundation of Shandong Province (2016JQB01015, 2017GSF18127). China Postdoctoral Science Foundation (2017M612213).

\section{Compliance with ethical standards}

Conflict of interest The authors declare that they have no conflict of interest.

\section{References}

1. Galluzzi L, Pedro JM, Demaria S, Formenti SC, Kroemer G. Activating autophagy to potentiate immunogenic chemotherapy and radiation therapy. Nat Rev Clin Oncol. 2016;14:247-58. 
2. Shimizu S, Kanaseki T, Mizushima N, Mizuta T, ArakawaKobayashi S, Thompson CB, et al. Role of Bcl-2 family proteins in a non-apoptotic programmed cell death dependent on autophagy genes. Nat Cell Biol. 2004;6:1221-8.

3. Yamamoto S, Sawada K, Shimomura H, Kawamura K, James TN. On the nature of cell death during remodeling of hypertrophied human myocardium. J Mol Cell Cardiol. 2000;32:161-75.

4. Tanaka Y, Guhde G, Suter A, Eskelinen EL, Hartmann D, Lullmann-Rauch $\mathrm{R}$, et al. Accumulation of autophagic vacuoles and cardiomyopathy in LAMP-2-deficient mice. Nature. 2000;406:902-6.

5. Maejima Y, Kyoi S, Zhai P, Liu T, Li H, Ivessa A, et al. Mst1 inhibits autophagy by promoting the interaction between Beclin1 and Bcl-2. Nat Med. 2013;19:1478-88.

6. Memczak S, Jens M, Elefsinioti A, Torti F, Krueger J, Rybak A, et al. Circular RNAs are a large class of animal RNAs with regulatory potency. Nature. 2013;495:333-8.

7. Guo JU, Agarwal V, Guo H, Bartel DP. Expanded identification and characterization of mammalian circular RNAs. Genome Biol. 2014;15:409.

8. Jeck WR, Sorrentino JA, Wang K, Slevin MK, Burd CE, Liu J, et al. Circular RNAs are abundant, conserved, and associated with ALU repeats. RNA (New Y, NY). 2013;19:141-57.

9. Chao CW, Chan DC, Kuo A, Leder P. The mouse formin (Fmn) gene: abundant circular RNA transcripts and gene-targeted deletion analysis. Mol Med (Camb, Mass). 1998;4:614-28.

10. Hansen TB, Jensen TI, Clausen BH, Bramsen JB, Finsen B, Damgaard CK, et al. Natural RNA circles function as efficient microRNA sponges. Nature. 2013;495:384-8.

11. Boeckel JN, Jae N, Heumuller AW, Chen W, Boon RA, Stellos K, et al. Identification and characterization of hypoxia-regulated endothelial circular RNA. Circ Res. 2015;117:884-90.

12. AbouHaidar MG, Venkataraman S, Golshani A, Liu B, Ahmad T. Novel coding, translation, and gene expression of a replicating covalently closed circular RNA of 220 nt. Proc Natl Acad Sci USA. 2014;111:14542-7.

13. Cherra SJ 3rd, Dagda RK, Tandon A, Chu CT. Mitochondrial autophagy as a compensatory response to PINK1 deficiency. Autophagy. 2009;5:1213-4.

14. Okatsu K, Kimura M, Oka T, Tanaka K, Matsuda N. Unconventional PINK1 localization to the outer membrane of depolarized mitochondria drives Parkin recruitment. J Cell Sci. 2015;128:964-78.

15. Sekine S, Youle RJ. PINK1 import regulation; a fine system to convey mitochondrial stress to the cytosol. BMC Biol. 2018;16:2.

16. Clark IE, Dodson MW, Jiang C, Cao JH, Huh JR, Seol JH, et al. Drosophila pink1 is required for mitochondrial function and interacts genetically with parkin. Nature. 2006;441:1162-6.

17. Zhou C, Huang Y, Shao Y, May J, Prou D, Perier C, et al. The kinase domain of mitochondrial PINK1 faces the cytoplasm. Proc Natl Acad Sci USA. 2008;105:12022-7.

18. Chen Y, Dorn GW 2nd. PINK1-phosphorylated mitofusin 2 is a Parkin receptor for culling damaged mitochondria. Science (New York, NY). 2013;340:471-5.

19. Lazarou M, Sliter DA, Kane LA, Sarraf SA, Wang C, Burman JL, et al. The ubiquitin kinase PINK1 recruits autophagy receptors to induce mitophagy. Nature. 2015;524:309-14.

20. Lai YC, Kondapalli C, Lehneck R, Procter JB, Dill BD, Woodroof HI, et al. Phosphoproteomic screening identifies Rab GTPases as novel downstream targets of PINK1. EMBO J. 2015;34:2840-61.

21. Wang X, Winter D, Ashrafi G, Schlehe J, Wong YL, Selkoe D, et al. PINK1 and Parkin target Miro for phosphorylation and degradation to arrest mitochondrial motility. Cell. 2011; 147:893-906.
22. Pridgeon JW, Olzmann JA, Chin LS, Li L. PINK1 protects against oxidative stress by phosphorylating mitochondrial chaperone TRAP1. PLoS Biol. 2007;5:e172.

23. Morais VA, Haddad D, Craessaerts K, De Bock PJ, Swerts J, Vilain S, et al. PINK1 loss-of-function mutations affect mitochondrial complex I activity via NdufA10 ubiquinone uncoupling. Science (New York, NY). 2014;344:203-7.

24. Lee Y, Stevens DA, Kang SU, Jiang H, Lee YI, Ko HS, et al. PINK1 primes Parkin-mediated ubiquitination of PARIS in dopaminergic neuronal survival. Cell Rep. 2017;18:918-32.

25. Plun-Favreau H, Klupsch K, Moisoi N, Gandhi S, Kjaer S, Frith $\mathrm{D}$, et al. The mitochondrial protease $\mathrm{HtrA} 2$ is regulated by Parkinson's disease-associated kinase PINK1. Nat Cell Biol. 2007;9:1243-52.

26. Valente EM, Abou-Sleiman PM, Caputo V, Muqit MM, Harvey K, Gispert S, et al. Hereditary early-onset Parkinson's disease caused by mutations in PINK1. Science (New York, NY). 2004;304:1158-60.

27. Nakajima A, Kataoka K, Hong M, Sakaguchi M, Huh NH. BRPK, a novel protein kinase showing increased expression in mouse cancer cell lines with higher metastatic potential. Cancer Lett. 2003;201:195-201.

28. Billia F, Hauck L, Konecny F, Rao V, Shen J, Mak TW. PTENinducible kinase 1 (PINK1)/Park6 is indispensable for normal heart function. Proc Natl Acad Sci USA. 2011;108:9572-7.

29. Rougerie P, Largeteau Q, Megrelis L, Carrette F, Lejeune T, Toffali $\mathrm{L}$, et al. Fam65b is a new transcriptional target of FOXO1 that regulates RhoA signaling for $\mathrm{T}$ lymphocyte migration. $\mathrm{J}$ Immunol (Baltimore, MD: 1950). 2013;190:748-55.

30. Diaz-Horta O, Subasioglu-Uzak A, Grati M, DeSmidt A, Foster J 2nd, Cao L, et al. FAM65B is a membrane-associated protein of hair cell stereocilia required for hearing. Proc Natl Acad Sci USA. 2014;111:9864-8.

31. Balasubramanian A, Kawahara G, Gupta VA, Rozkalne A, Beauvais A, Kunkel LM, et al. Fam65b is important for formation of the HDAC6-dysferlin protein complex during myogenic cell differentiation. FASEB J. 2014;28:2955-69.

32. Dakour J, Li H, Morrish DW. PL48: a novel gene associated with cytotrophoblast and lineage-specific HL-60 cell differentiation. Gene. 1997;185:153-7.

33. Froehlich J, Versapuech M, Megrelis L, Largeteau Q, Meunier S, Tanchot C, et al. FAM65B controls the proliferation of transformed and primary T cells. Oncotarget. 2016;7:63215-25.

34. Gao K, Tang W, Li Y, Zhang P, Wang D, Yu L, et al. Frontsignal-dependent accumulation of the RHOA inhibitor FAM65B at leading edges polarizes neutrophils. $J$ Cell Sci. 2015;128:992-1000.

35. Tan WQ, Wang K, Lv DY, Li PF. Foxo3a inhibits cardiomyocyte hypertrophy through transactivating catalase. J Biol Chem. 2008;283:29730-9.

36. Pasman Z, Been MD, Garcia-Blanco MA. Exon circularization in mammalian nuclear extracts. RNA (New Y, NY). 1996; 2:603-10.

37. Li PF, Dietz R, von Harsdorf R. p53 regulates mitochondrial membrane potential through reactive oxygen species and induces cytochrome c-independent apoptosis blocked by Bcl-2. EMBO J. 1999;18:6027-36.

38. Wang K, Liu CY, Zhou LY, Wang JX, Wang M, Zhao B, et al. APF IncRNA regulates autophagy and myocardial infarction by targeting miR-188-3p. Nat Commun. 2015;6:6779.

39. Jin Y, Manabe T. High-efficiency protein extraction from polyacrylamide gels for molecular mass measurement by matrixassisted laser desorption/ionization-time of flight-mass spectrometry. Electrophoresis. 2005;26:1019-28. 
40. Shevchenko A, Tomas Henrik, sbreve JH, Olsen JV, Mann M. Ingel digestion for mass spectrometric characterization of proteins and proteomes. Nat Protoc. 2007;1:2856-60.

41. Kabeya Y, Mizushima N, Ueno T, Yamamoto A, Kirisako T, Noda T, et al. LC3, a mammalian homologue of yeast Apg8p, is localized in autophagosome membranes after processing. EMBO J. 2000;19:5720-8.

42. Lin Z, Murtaza I, Wang K, Jiao J, Gao J, Li PF. miR-23a functions downstream of NFATc3 to regulate cardiac hypertrophy. Proc Natl Acad Sci USA. 2009;106:12103-8.

43. Wang K, Zhou LY, Wang JX, Wang Y, Sun T, Zhao B, et al. E2F1-dependent miR-421 regulates mitochondrial fragmentation and myocardial infarction by targeting Pink1. Nat Commun. 2015;6:7619.

44. Zhou C, Huang Y, Shao Y, May J, Prou D, Perier C, et al. The kinase domain of mitochondrial PINK1 faces the cytoplasm. Proc Natl Acad Sci. 2008;105:12022-7.

45. Yoon S, Molloy MJ, Wu MP, Cowan DB, Gussoni E. C6ORF32 is upregulated during muscle cell differentiation and induces the formation of cellular filopodia. Dev Biol. 2007;301:70-81.

46. Huttlin EL, Jedrychowski MP, Elias JE, Goswami T, Rad R, Beausoleil SA, et al. A tissue-specific atlas of mouse protein phosphorylation and expression. Cell. 2010;143:1174-89.

47. Beilina A, Van Der Brug M, Ahmad R, Kesavapany S, Miller DW, Petsko GA, et al. Mutations in PTEN-induced putative kinase 1 associated with recessive parkinsonism have differential effects on protein stability. Proc Natl Acad Sci USA. 2005;102:5703-8.

48. Valentim L, Laurence KM, Townsend PA, Carroll CJ, Soond S, Scarabelli TM, et al. Urocortin inhibits Beclin1-mediated autophagic cell death in cardiac myocytes exposed to ischaemia/ reperfusion injury. J Mol Cell Cardiol. 2006;40:846-52.

49. Kostin S, Pool L, Elsasser A, Hein S, Drexler HC, Arnon E, et al. Myocytes die by multiple mechanisms in failing human hearts. Circ Res. 2003;92:715-24.

50. Liang XH, Jackson S, Seaman M, Brown K, Kempkes B, Hibshoosh $\mathrm{H}$, et al. Induction of autophagy and inhibition of tumorigenesis by beclin 1. Nature. 1999;402:672-6.

51. Holdt LM, Stahringer A, Sass K, Pichler G, Kulak NA, Wilfert W, Kohlmaier A, Herbst A, Northoff BH, Nicolaou A, Gabel G, Beutner F, Scholz M, Thiery J, Musunuru K, Krohn K. Circular non-coding rna anril modulates ribosomal rna maturation and atherosclerosis in humans. 2016;7:12429.

52. Zheng Q, Bao C, Guo W, Li S, Chen J, Chen B, et al. Circular RNA profiling reveals an abundant circHIPK3 that regulates cell growth by sponging multiple miRNAs. Nat Commun. 2016;7:11215.
53. Xie H, Ren X, Xin S, Lan X, Lu G, Lin Y, et al. Emerging roles of circRNA_001569 targeting miR-145 in the proliferation and invasion of colorectal cancer. Oncotarget. 2016;7:26680-91.

54. Ahmed I, Karedath T, Andrews SS, Al-Azwani IK, Mohamoud YA, Querleu D, et al. Altered expression pattern of circular RNAs in primary and metastatic sites of epithelial ovarian carcinoma. Oncotarget. 2016;7:36366-81.

55. Capel B, Swain A, Nicolis S, Hacker A, Walter M, Koopman P, et al. Circular transcripts of the testis-determining gene Sry in adult mouse testis. Cell. 1993;73:1019-30.

56. Li Z, Huang C, Bao C, Chen L, Lin M, Wang X, et al. Exon-intron circular RNAs regulate transcription in the nucleus. Nat Struct. 2015;22:256-64.

57. Rakovic A, Shurkewitsch K, Seibler P, Grunewald A, Zanon A, Hagenah $\mathrm{J}$, et al. Phosphatase and tensin homolog (PTEN)induced putative kinase 1 (PINK1)-dependent ubiquitination of endogenous Parkin attenuates mitophagy: study in human primary fibroblasts and induced pluripotent stem cell-derived neurons. J Biol Chem. 2013;288:2223-37.

58. Dagda RK, Cherra SJ 3rd, Kulich SM, Tandon A, Park D, et al. Loss of PINK1 function promotes mitophagy through effects on oxidative stress and mitochondrial fission. $\mathrm{J}$ Biol Chem. 2009;284:13843-55.

59. Gomez-Sanchez R, Yakhine-Diop SM, Bravo-San Pedro JM, Pizarro-Estrella E, Rodriguez-Arribas M, Climent V, et al. PINK1 deficiency enhances autophagy and mitophagy induction. Mol Cell Oncol. 2016;3:e1046579.

60. Cui T, Fan C, Gu L, Gao H, Liu Q, Zhang T, et al. Silencing of PINK1 induces mitophagy via mitochondrial permeability transition in dopaminergic MN9D cells. Brain Res. 2011; 1394:1-13.

61. Qi Z, Yang W, Liu Y, Cui T, Gao H, Duan C, et al. Loss of PINK1 function decreases PP2A activity and promotes autophagy in dopaminergic cells and a murine model. Neurochem Int. 2011;59:572-81.

62. Petit A, Kawarai T, Paitel E, Sanjo N, Maj M, Scheid M, et al. Wild-type PINK1 prevents basal and induced neuronal apoptosis, a protective effect abrogated by Parkinson disease-related mutations. J Biol Chem. 2005;280:34025-32.

63. Fedorowicz MA, de Vries-Schneider RL, Rub C, Becker D, Huang Y, Zhou C, et al. Cytosolic cleaved PINK1 represses Parkin translocation to mitochondria and mitophagy. EMBO Rep. 2014;15:86-93.

64. Megrelis L, Ghoul EE, Moalli F, Versapuech M, Cassim S, Ruef N, et al. Fam65b phosphorylation relieves tonic RhoA inhibition during $\mathrm{T}$ cell migration. Front Immunol. 2018;9: 2001. 